\title{
Local Rules for Pentagonal Quasi-Crystals
}

\author{
Le Tu Quoc Thang \\ Department of Mathematics, SUNY at Buffalo, \\ Buffalo, NY 14214, USA \\ Communicated by Marjorie Senechal
}

\begin{abstract}
The existence of different kinds of local rules is established for many sets of pentagonal quasi-crystal tilings. For each $t \in \mathbb{R}$ there is a set $\overline{\mathscr{T}}_{t}$ of pentagonal tilings of the same local isomorphism class; the case $t=0$ corresponds to the Penrose tilings. It is proved that the set $\overline{\mathscr{T}}_{t}$ admits a local rule which does not involve any colorings (or markings, decorations) if and only if $t=m+n \tau$. In other words, this set of tilings is totally characterized by patches of some finite radius, or $r$-maps. When $t=(m+n \sqrt{5}) / q$ the set $\overline{\mathscr{T}}_{t}$ admits a local rule which involves colorings. For the set of Penrose tilings the construction here leads exactly to the Penrose matching rules. Local rules for the case $t=\frac{1}{2}$ are presented.
\end{abstract}

\section{Introduction}

The aim of this paper is to find local rules (or matching rules) which force tilings to belong to a specific set of tilings. The tilings considered in this paper are the sets of pentagonal quasi-crystal tilings, also sometimes called quasi-periodic tilings with fivefold symmetry, or generalized Penrose two-dimensional tilings. These tilings have received a great deal of attention among mathematicians and physicists (see [dB1], [KP], and [IS]). The method used in this paper can be applied to other classes of quasi-periodic tilings of two or higher dimensions. The infinitely many sets of pentagonal quasi-crystals are parametrized by $t \in \mathbb{R}$; we denote them by $\overline{\mathscr{T}}_{t}$. The exact definition of these tilings are given in Section 1. They have many nice properties, and they are quasi-periodic by perhaps all the known definitions of quasi-periodicity. For each $t$ the set $\widetilde{\mathscr{T}}_{t}$ consists of tilings of the same local isomorphism class: every finite part of a tiling in $\overline{\mathscr{T}}_{t}$ can be found in any other tiling in $\overline{\mathscr{T}}_{t}$. All these tilings are obtained by the strip projection method [dB1], [KP]. 


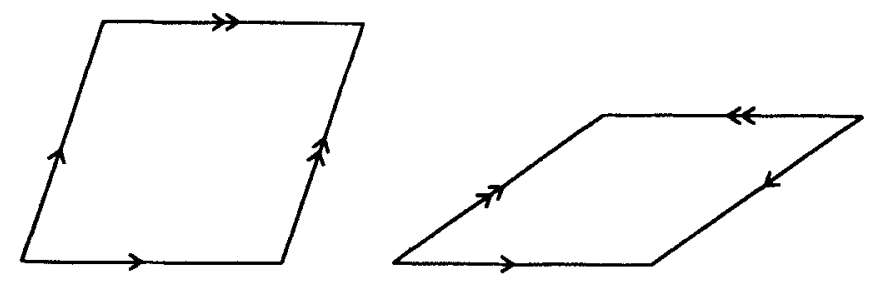

(a)
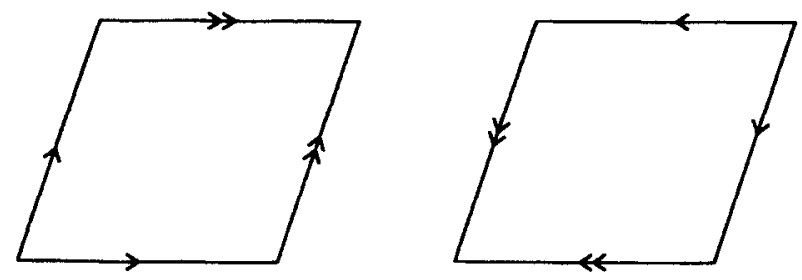

(b)

Fig. 1. The Penrose local rule.

The set of tilings $\overline{\mathscr{T}}_{0}$ was discovered by Penrose; it is perhaps the best known among nonperiodic tilings. As an example we give here the local rule which forces tilings to belong to $\overline{\mathscr{F}}_{0}$ (see [P] and [dB1]). Let us consider two rhombs whose acute angles are $\pi / 5$ and $2 \pi / 5$, and whose edges are of the same length. The edges are equipped with arrows as in Fig. 1(a). A tiling of a two-dimensional plane by these "arrowed" rhombs is said to satisfy the Penrose local rule if every edge of this tiling has a definite arrow, that is, the arrows of an edge coming from two rhombs incident to this edge are coincident.

Suppose $T$ is a tiling of the plane by copies of these rhombs, without any arrows. We say that $T$ satisfies the Penrose local rule if there is a way to put arrows on edges of rhombs of $T$ such that the arrowed tiling satisfies the Penrose local rule. A fundamental result of De Bruijn states that the set $\overline{\mathscr{T}}_{0}$ is the set of all tilings satisfying the Penrose local rule.

The main question of this paper is whether there are similar local nules for other sets $\overline{\mathscr{T}_{t}}$ of pentagonal quasi-crystals. This question is also studied in [KP] and [IS].

Note that a "local rule," in some sense, contains information in a local finite radius. It is far from trivial to decide when a local rule forces tilings, say, to be nonperiodic, or quasi-periodic, or to belong to a specific set consisting of tilings of the same isomorphism class. The question whether a set of (usually quasi-periodic in some sense) tilings admits a local rule also has importance for physics. It seems that only such sets of tilings can serve as model for real quasi-crystals such as those discovered in 1984 (see discussions in [K], [Lev], and [LPS2]). The similar question of finding a set of prototiles such that every tiling by these prototiles must be aperiodic, or, more difficult, quasi-periodic in some sense but not periodic, seems interesting and has been investigated by many authors. 
There are two types of local rules discussed in this paper; their definition are given in Section 1. The main difference between them is the first one does not involve any coloring (marking, decorations) while the second one does.

Our chief results can be summarized as follows. First we prove that if $t=(\mathrm{m}+$ $n \sqrt{5}) / q$, then the set $\overline{\mathscr{F}_{t}}$ admits a local rule of type 2 . Furthermore, this local rule consists of patches containing only two neighboring tiles. The case $t=0$ corresponds to the Penrose tilings, and the construction of local rules here leads exactly to the Penrose local rule. Hence De Bruijn's result can be regarded as a special case of our results. We prove that if $t=m+n \tau$ where $\tau=(1+\sqrt{5}) / 2$, then this local rule can be realized by a local rule of type 1 ; hence $\overline{\mathscr{T}}_{t}$ admits a local rule of type 1 . It follows from a result of Ingersent and Steinhardt [IS] that $t=m+n \tau$ is also a necessary condition for the existence of local rules of type 1 . Hence we have a criterion for the existence of local rules of type 1. As an illustration, we describe the local rule (of type 2) for the case $t=\frac{1}{2}$. Actually, we have to refine the method so that we could get a simple local rule. This local rule is the Kleman-Pavlovitch local rule [KP] enhanced with some condition on the vertex which is very similar to the Ammann and Socolar local rules for eight-fold and twelve-fold tilings.

The existence of local rules of type 2 has been established for sets of quasi-crystals based on quadratic irrationality of any dimensions [LPS1], [LPS2]. The eight-fold two-dimensional and icosahedral three-dimensional tilings are special cases of these results.

We would like to emphasize the fact that many sets of tilings that do not admit local rules of the first type do admit local rules of the second type. An example is the set of tilings with eight-fold symmetry (see [B], [dB2], [Le1], and [So1]), or any set $\overline{\mathscr{T}}_{t}$ with $t=(m+n \sqrt{5}) / q$ where $t$ is not of the form $m+n \tau$. For those sets of tilings, the problem of coloring a tiling to get a colored tiling satisfying the local rule of type 2 is not a local problem: one cannot decide how to color a tile by inspecting a bounded region around this tile. As observed by Senechal [Se] there is a tiling in $\overline{\mathscr{T}}_{t}$ which can be colored in two different ways. This is very different from the set of penrose tilings. For this class, we can decide how to arrow the edges of a tile by inspection around this tile within the radius 2 ; and every tiling can be arrowed in a unique way.

Here is a very rough sketch of the proof of the existence of a type 2 local rule. By the cut method, there is a periodic tiling $\mathscr{O}_{t}$ of $\mathbb{R}^{4}$ whose tiles are prisms with the base parallel to a two-dimensional plane $\mathbf{E}$; and every tiling in $\overline{\mathscr{T}}_{t}$ is obtained as a slice of $\mathscr{O}_{t}$ by a two-dimensional plane parallel to $\mathbf{E}$. In other words, a tiling in $\overline{\mathscr{T}}_{t}$ is the projection of the tiles of $\mathscr{O}_{t}$ which meet a fixed 2-plane parallel to E. Suppose $T$ is a tiling such that every pair of neighboring tiles of $T$ is a translate of a pair of neighboring tiles of some tiling in $\overline{\mathscr{F}}_{t}$ (i.e., $T$ satisfies some special local rule). Then we can lift $T$ onto $\mathscr{O}_{t}$ : we can choose a collection of tiles of $\mathscr{O}_{t}$ which project onto tiles of $T$. The lift has an important property: for every pair of neighboring members in this collection, there is a plane parallel to $\mathbf{E}$ which meets the interior of both of them. In some cases this is enough to prove that:

( $\star$ ) There is a plane parallel to $\mathbf{E}$ meeting all the members of this collection.

This means $T$ is defined by a slice of $\mathscr{O}_{t}$, i.e., $T$ belongs to $\mathscr{F}_{t}$. 
Technically the proof of $(\star)$ is rather complicated. First we have to prove that our local rule is a weak local rule in the sense of Levitov [Lev]. Actually this was established in a general setting in [LPS2]; we give a proof for our case in the Appendix. Then we have to study the boundary of the tiles in $\mathscr{\theta}_{t}$ and in general we have to replace $\sigma_{t}$ by its "refinement" so that the boundary matches some conditions. This step corresponds to the coloring of the local rules. The method used here can be easily generalized for other sets of quasi-periodic tilings obtained by the projection method. There is no specific property of the case investigated here that is used for the proof, except that in this case, due to some symmetry, we can reduce the number of verifications. The general result of the existence of type 2 local rules (local rules involving coloring) is formulated in Section 7, Theorem 7.1.

To establish the existence of a type 1 local rule we do need some specific property of the case under investigation. Actually we prove that if $t=m+n \tau$, there is some finite radius such that the coloring of a tile is uniquely defined by the configuration of the tiling inside the disk of this radius around the tile. It also follows from the proof that the radius is linearly dependent on $n$.

The paper is organized as follows. In Section 1 we introduce definitions and preliminary facts. In Section 2 some facts about the cut method are recalled. We follow the paper [ODK]. In Section 3 we give the proof of the existence of a local rule of type 2 for the case $t=0$; the proof is readily generalized in Section 4 for the case $t=(m+n \sqrt{5}) / q$. We also prove that the local rule obtained for $t=0$ is equivalent to the Penrose local rule. Section 5 is devoted to the case $t=m+n \tau$; the existence of a local rule of type 1 is proved. In Section 6 we prove a technical result used in Sections 3 and 4 . Section 7 contains the example $t=\frac{1}{2}$, some generalizations, and concluding remarks. In the Appendix we prove a generalization of a result of Levitov concerning the weak local rule.

\section{Definitions and Preliminary Facts}

For technical convenience some of our definitions (tilings, prototiles, etc.) are more special than is generally the case. In particular, we use translational congruence instead of the usual congruence which involves rotations and reflections (our results can be easily reformulated in terms of the usual congruence).

\subsection{On Tilings and Local Rules}

Two subsets of $\mathbb{R}^{k}$ are called congruent if the second is a translate of the first. We always distinguish between two congruent polyhedra.

A tiling of $\mathbb{R}^{k}$ is a family of $k$-dimensional polyhedra, called the tiles of this tiling, which covers $\mathbb{R}^{k}$ without overlaps (that is, the interiors of two different tiles have empty intersection). In this paper, except in the case of the oblique periodic tilings 
which appear later, all tilings are assumed to be face-to-face type, i.e., the intersection of every two tiles is a common facet of lower dimension, if not empty. A vertex, edge, facet, etc., of a tiling is, respectively, any vertex, edge, facet, etc., of one of its tiles. For a given tiling the translation classes of tiles are called the prototiles of this tiling. All the tilings encountered in this paper are tilings of some Euclidean space with a fixed origin $\mathbf{0}$. We use the following definition of convergence of tilings (compare [Ra1] and [Ro]).

Definition. A sequence of tilings $T_{1}, T_{2}, \ldots$, of $\mathbb{B}^{k}$ converges to a tiling $T$ if, for every $r>0$, there is a natural number $N$ such that, for $i>N$, the tiling $T_{i}$ coincides with $T$ inside the disk $U_{r}$ with center at $\mathbf{0}$ and radius $r$.

We define the closure $\overline{\mathscr{U}}$ of a set $\mathscr{U}$ of tilings as the set of all the limits of sequences of tilings belonging to $\mathscr{U}$. A set of tilings is closed if it is coincident with its closure.

An r-map of a tiling $T$ at a vertex $v$ is the collection of all the tiles lying inside the ball of radius $r$ centered at $v$. More generally, an r-map is any $r$-map of any tiling at any vertex. Two $r$-maps are congruent if the second is a translate of the first.

Definition. A local rule of type 1 of radius $r$ is a finite set of $r$-maps. A tiling $T$ satisfies a local rule $\mathscr{A}$ of type 1 of radius $r$ if every $r$-map of $T$ is congruent to one from $\mathscr{A}$.

By "a local rule of type 1" we mean a local rule of type 1 of some radius.

\subsection{Coloring}

$A$ colored polyhedron is a pair $(P, j)$ where $P$ is a polyhedron and $j$ is an arbitrary element, called the color of this polyhedron. Two colored polyhedra are congruent if their colors are the same and the second is a translate of the first. A colored tiling (resp. a colored $r$-map) is a tiling (resp. $r$-map) whose tiles are colored polyhedra. Two colored $r$-maps are congruent if the second is a translate of the first and colors of the corresponding tiles are coincident. As in the previous section, we can define colored $r$-maps of colored tilings, limits of sequences of colored tilings, and closures of sets of colored tilings.

The following definition of a local rule of type 2 is introduced only for the two-dimensional case.

An edge-configuration is a collection of two colored polygons having a full common edge. Two edge-configurations are congruent if the second is a translate of the first and the corresponding colors are the same. For a colored tiling of $\mathbb{R}^{2}$ the edge-configuration of an edge is the pair of colored tiles incident to this edge.

Definition. A local rule of type 2 is a finite set of edge-configurations. A colored tiling satisfies a local rule of type $2 \mathscr{B}$ if the edge-configuration of every its edge is 
congruent to one from $\mathscr{B}$. A noncolored tiling satisfies this local rule if it can be colored to become a colored tiling satisfying this local rule.

Definition. A set of noncolored tilings admits a local rule of any type if it is the set of all tilings satisfying this local rule.

It is easy to see that if a set of tilings admits a local rule of any type, then this set is closed.

\section{Remarks.}

1. The coloring makes the number of prototiles become larger. For example the two Penrose tiles in Fig. 1(b) are not congruent.

2. The Penrose local rule is a local rule of type 2 . All the proofs for the absence of local rules (maybe under some restrictions) in [B], [L], [Le1], and [IS] are only for local rules of type 1.

3. In [Le1], [Le2], and [LPS2] local rules of type 1 are called simply "local rule"; local rules of type 2 are special cases of "local rules with coloring" there. Danzer [D2] calls local rules of type 2 "strictly local matching rules." Our definition of a local rule of type 1 corresponds to the $r$-rule of Levitov [Lev].

\subsection{The Superspace $\mathbb{R}^{5}$}

In the Euclidean space $\mathbb{R}^{5}$ with origin 0 we fix a standard base $\varepsilon_{0}, \varepsilon_{1}, \varepsilon_{2}, \varepsilon_{3}, \varepsilon_{4}$. Let $\mathbb{Z}^{5}$ be the lattice of integer points, and let $\mathbb{Q} \sqrt{5}$ be the set of all numbers of the form $a+b \sqrt{5}$, where $a$ and $b$ are rational numbers. If $x$ is a point in $(\mathbb{Q} \sqrt{5})^{5}$, then $x=y+z \sqrt{5}$ where $y$ and $z$ are rational points in $\mathbb{R}^{5}$, i.e., points with rational coordinates. The point $y-z \sqrt{5}$ is called the conjugate of $x$. If $X$ and $Y$ are subsets of $\mathbb{R}^{5}$ put $X+Y=\{x+y \mid x \in X, y \in Y\}$, and $-X=\{-x \mid x \in X\}$.

A subspace (as a vector space) of $\mathbb{R}^{5}$ is called a homogeneous plane and its translates are called planes; an $n$-plane is a plane of dimension $n$. A plane $F$ is called rational if $F$ is homogeneous and spanned by vectors with rational coordinates.

Consider the action of cyclic group $\mathbb{Z}_{5}=\left\langle g \mid g^{5}=1\right\rangle$ on $\mathbb{R}^{5}$ by cyclic permutation of the base: $g\left(\varepsilon_{i}\right)=\left(\varepsilon_{i+1}\right)(\bmod 5)$. The space $\mathbb{R}^{5}$ decomposes into three invariant subspaces $\mathbf{E}, \overline{\mathbf{E}}$, and $\Delta$. Here $\Delta$ is the one-dimensional subspace spanned by $\delta=\left(\varepsilon_{0}+\varepsilon_{1}+\varepsilon_{2}+\varepsilon_{3}+\varepsilon_{4}\right) / 5, \mathbf{E}$ is the 2-plane spanned by two vectors with coordinates $(4, \sqrt{5}-1,-\sqrt{5}-1,-\sqrt{5}-1, \sqrt{5}-1)$ and $(\sqrt{5}-1,4, \sqrt{5}-$ $1,-\sqrt{5}-1,-\sqrt{5}-1), \overline{\mathbf{E}}$ is the 2 -plane spanned by the conjugates of these two vectors. The element $g$ acts on $\mathbf{E}$ as rotation by $2 \pi / 5$, on $\overline{\mathbf{E}}$ as rotation by $4 \pi / 5$, and on $\Delta$ as the identity.

Putting $\mathbf{E}^{\perp}=\mathbf{E}+\Delta, \mathbb{R}^{5}=\mathbf{E} \oplus \overline{\mathbf{E}} \oplus \Delta=\mathbf{E} \oplus \mathbf{E}^{\perp}$. Let $\mathbf{p}, \overline{\mathbf{p}}, \mathbf{p}^{\perp}, \mathbf{p}_{\Delta}$ be, respectively, the projectors of $\mathbb{R}^{5}$ on $\mathbf{E}, \overline{\mathbf{E}}, \mathbf{E}^{\perp}, \Delta$. We define $e_{i}=\mathbf{p}\left(\varepsilon_{i}\right), e_{i}^{\perp}=\mathbf{p}^{\perp}\left(\varepsilon_{i}\right)$, and $\bar{e}_{i}=\overline{\mathbf{p}}\left(\varepsilon_{i}\right)$. The projection of every of $\varepsilon_{i}$ on $\Delta$ is $\delta$. Hence $\mathbf{p}_{\Delta}(\xi)$ is a multiple of $\delta$ for every $\xi \in \mathbb{Z}^{5}$. 
For a real number $t$ let $\overline{\mathbf{E}}_{t}=\overline{\mathbf{E}}+t \delta$. Denote the 4-plane $\mathbf{E}+\overline{\mathbf{E}}_{t}$ by $\mathbb{R}_{t}^{4}$. Then $\mathbb{R}^{5}=\bigcup_{t \in \mathbb{R}} \mathbb{R}_{t}^{4}$. For example, $\mathbb{R}_{0}^{4}$ is the rational 4-plane $\mathbf{E} \oplus \overline{\mathbf{E}}$; it is the set of all points whose coordinates sum to 0 . Let $\Lambda=\mathbb{R}_{0}^{4} \cap \mathbb{Z}^{5}$ be the set of all integer points lying in $\mathbb{R}_{0}^{4}$; it is the lattice generated by $\varepsilon_{0}-\varepsilon_{1}, \varepsilon_{1}-\varepsilon_{2}, \varepsilon_{2}-\varepsilon_{3}, \varepsilon_{3}-\varepsilon_{4}$.

The following is easy to check.

Proposition 1.1. $\mathbf{p}(\xi)=\mathbf{p}(\eta)$, where $\xi, \eta \in \mathbb{Z}^{5}$, if and only if $\xi-\eta$ is a multiple of $5 \delta$.

Definition. Let $x=\mathbf{p}(\xi)$ where $\xi=\sum_{i=0}^{4} n_{i} \varepsilon_{i}, n_{i} \in \mathbb{Z}$, is an integer point. The $\operatorname{index}(x)$ is the remainder of the sum $\sum_{i=0}^{4} n_{i}$ modulo 5 (see also [dB1] and [KP]).

It follows from Proposition 1.1 that the index is well defined; it is defined only for points in $\mathbf{p}\left(\mathbb{Z}^{5}\right)$. For $x, y \in \mathbf{p}\left(\mathbb{Z}^{5}\right)$ we have $\operatorname{index}(x+y) \equiv \operatorname{index}(x)+\operatorname{index}(y)$ $(\bmod 5)$.

The projection $\mathbf{p}\left(\mathbb{Z}^{5}\right)$ on $\mathbf{E}$ is a dense $\mathbb{Z}$-module generated by five vectors $e_{1}, \ldots, e_{5}$ which point to the vertices of a regular pentagon (see Fig. 2). The set $\mathbf{p}^{\perp}\left(\mathbb{Z}^{5}\right)$ is not dense in $\mathbf{E}^{\perp}$ but is contained and dense in the union of parallel and equidistant 2-planes $\overline{\mathbf{E}}+k \delta$, where $k \in \mathbb{Z}$.

The following is also easy to check.

\section{Proposition 1.2.}

(a) If $\mathbf{p}_{\Delta}(\xi)=m \delta$, then $m \in \mathbb{Z}$ and $m \equiv \operatorname{index}(\mathbf{p}(\xi))(\bmod 5)$.

(b) If $\mathbf{p}_{\Delta}(\xi)=\mathbf{0}$, where $\xi \in \mathbb{Z}^{5}$, then $\xi$ belongs to $\Lambda$ (recall that $\Lambda=\mathbb{R}_{0}^{4} \cap \mathbb{Z}^{5}$ ).

(c) If two rational points $\xi$, $\xi^{\prime}$ have the same projection on $\mathbf{E}^{\perp}$, then $\xi=\xi^{\prime}$.

(d) If a rational $k$-plane $F$ contains $\mathbf{E}$, then it contains $\mathbb{R}_{0}^{4}=\mathbf{E} \oplus \overline{\mathbf{E}}$.

\subsection{The Strip Projection Method}

If $v_{1}, v_{2}, \ldots, v_{k}$ are vectors of $\mathbb{R}^{5}$ let $\operatorname{Pol}\left(v_{1}, \ldots, v_{k}\right)$ be the polyhedron:

$$
\operatorname{Pol}\left(v_{1}, \ldots, v_{k}\right)=\left\{\sum_{i=1}^{i=k} \lambda_{i} v_{i}, \lambda_{i} \in[0,1]\right\} \text {. }
$$
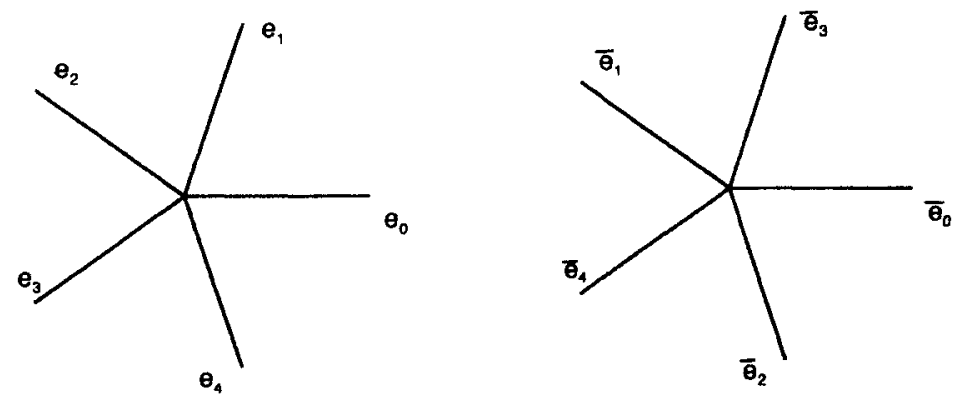

Fig. 2. The projections of the base. 
The set $\gamma=\operatorname{Pol}\left(\varepsilon_{0}, \varepsilon_{1}, \varepsilon_{2}, \varepsilon_{3}, \varepsilon_{4}\right)$ is the unit hypercube of the lattice $\mathbb{Z}^{5}$. The set $\operatorname{Pol}\left(\varepsilon_{i}, \varepsilon_{j}\right)$ (for $\left.0 \leq i<j \leq 4\right)$ is called a 2-facet of $\gamma$ and its translates $\xi+\operatorname{Pol}\left(\varepsilon_{i}, \varepsilon_{j}\right)$, where $\xi \in \mathbb{Z}^{5}$, are called the 2 -facets of the lattice $\mathbb{Z}^{5}$.

The set of all pairs $(i, j)$ with $0 \leq i<j \leq 4$ is denoted by $M$. For convenience a rhomb in this paper always refers to a translate of one of ten $P_{I}=\operatorname{Pol}\left(e_{i}, e_{j}\right)$ with $I=(i, j) \in M$. Up to rotation there are only two rhombs from the ten $P_{i}$, they are shown in Fig. 1(a).

For every $\alpha \in \mathbf{E}^{\perp}$ let $\mathbf{S}_{\alpha}$ be the strip defined by shifting the unit hypercube $\gamma$ along the 2-plane $\mathbf{E}+\alpha: \mathbf{S}_{\alpha}=\mathbf{E}+\alpha+\gamma, \alpha \in \mathbf{E}^{\perp}$.

Definition. $\alpha \in \mathbf{E}^{\perp}$ is regular if the boundary of the strip $\mathbf{S}_{\alpha}$ does not contain any integer point. Otherwise $\alpha$ is singular.

The following facts are fundamental in the strip projection method. For regular $\alpha$ the union of all the 2 -facets of the lattice $\mathbb{Z}^{5}$ lying in the strip $\mathbf{S}_{\alpha}$ is a two-dimensional continuous surface. This surface contains all the vertices of the lattice $\mathbb{Z}^{5}$ falling inside $\mathbf{S}_{\alpha}$ and has an obvious polyhedral structure. By projecting this polyhedral structure along $\mathbf{E}^{\perp}$ onto $\mathbf{E}$ we get a tiling $T_{\alpha}$ of $\mathbf{E}$. Note that there are no overlaps: the restriction of $\mathbf{p}$ to this surface is one-to-one. The prototiles are the ten rhombs $P_{I}, I \in M$. This method of obtaining the tilings $T_{\alpha}$ is the strip projection method, applied to our setting (see [dB1], [GR], and [ODK]).

Denote by $\mathscr{T}$ (resp. $\mathscr{T}_{t}$ ) the set of all tilings $T_{\alpha}$ for regular $\alpha \in \mathbf{E}^{\perp}$ (resp. $\alpha \in \overline{\mathbf{E}}_{t}$ ) and their translates.

Definition. A tiling of the closure $\overline{\mathscr{T}}$ of $\mathscr{T}$ is called a pentagonal quasi-crystal.

Of course if $\alpha \in \overline{\mathbf{E}}_{t}$ is regular, then any translate of $T_{\alpha}$ is in $\overline{\mathscr{T}}_{t}$, but other tilings in $\overline{\mathscr{F}}_{t}$ exist as well. We describe such tilings in Section 2.5 .

Two important properties of pentagonal quasi-crystals are [dB1], [KP], [ODK]:

\section{Proposition 1.3.}

(a) Local isomorphism: for every two tilings in $\overline{\mathscr{T}}_{t}$, for every $r>0$, every $r$-map of the first tiling is congruent to an r-map of the second.

(b) Symmetry: if $T$ is a tiling of $\overline{\mathscr{T}}_{t}$, then the rotation of $T$ by $2 \pi / 5$ also belongs to $\overline{\mathscr{T}}_{\boldsymbol{t}}$.

Remark. Some authors consider only regular cases, that is, the sets $\mathscr{T}_{t}$ and $\mathscr{F}$ but not their closures. However, as argued above, the question of finding a local rule for $\mathscr{T}_{t}, \mathscr{T}$ is not relevant, because these sets are not closed. In each set of $\overline{\mathscr{T}}_{t}, \overline{\mathscr{T}}$ there are two different tilings coincident in a half-plane. 


\section{The Cut Method}

\subsection{The Cut Method in the Superspace}

A set $X$ in $\mathbb{R}^{5}$ is called a prism if $X=\mathbf{p}(X)+\mathbf{p}^{\perp}(X)$. If $X$ and $Y$ are prisms, then their intersection is also a prism, and we have the nice formula

$$
X \cap Y=[\mathbf{p}(X) \cap \mathbf{p}(Y)]+\left[\mathbf{p}^{\perp}(X) \cap \mathbf{p}^{\perp}(Y)\right]
$$

This makes it easy to study the intersection of two prisms.

Definition. Suppose $X$ is a prism such that $p^{\perp}(X)$ is a polyhedron. We define the parallel boundary of $X$ as

$$
\mathbf{p}(X)+\partial\left(\mathbf{p}^{\perp}(\mathbf{X})\right)
$$

where $\partial\left(\mathbf{p}^{\perp}(X)\right)$ is the boundary of $\mathbf{p}^{\perp}(X)$.

The parallel boundary of a prism is a part of its boundary.

For $I=\left(i_{0}, i_{1}\right) \in M$ recall that $P_{I}$ is the rhomb $\operatorname{Pol}\left(e_{i_{0}}, e_{i_{1}}\right)$. Let

$$
P_{I}^{\perp}=-\operatorname{Pol}\left(e_{j_{0}}^{\perp}, e_{j_{1}}^{\perp}, e_{j_{2}}^{\perp}\right)
$$

where $\left(i_{0}, i_{1}, j_{0}, j_{1}, j_{2}\right)$ is a permutation of $(0,1,2,3,4)$. Put $C_{I}=P_{I}+P_{I}^{\perp}$; it is a prism.

Consider the family $\mathscr{O}$ consisting of all prisms of the form $C_{I}+\xi$ for $I \in M$ and $\xi \in \mathbb{Z}^{5}$. It is proved in [ODK] that this family covers $\mathbb{R}^{5}$ without any overlaps, i.e., it is a tiling of $\mathbb{R}^{5}$. The tiling $\mathscr{G}$ is not of face-to-face type and is invariant under translations by vectors of $\mathbb{Z}^{5}$. The parallel boundary $\mathbf{B}$ of $\mathscr{G}$, by definition, is the union of the parallel boundaries of all the tiles of $\mathscr{O}$. It is a cellular complex of dimension 4. If $\alpha \in \mathbf{E}^{\perp}$ is such that $\mathbf{E}+\alpha$ does not meet $\mathbf{B}$, then all the intersections of $\mathbf{E}+\alpha$ with tiels of 6 form a cover of $\mathbf{E}+\alpha$ without overlaps and define a tiling of $\mathbf{E}+\alpha$. Projecting along $\mathbf{E}^{\perp}$ we get a tiling of $\mathbf{E}$, called the tiling defined by $\mathscr{G}$ and $\alpha$. If $\mathbf{E}+\alpha$ meets $\mathbf{B}$, then all the intersections of $\mathbf{E}+\alpha$ with covers $\mathbf{E}+\alpha$ with overlaps. The fundamental result of [ODK] can be stated as follows.

Theorem 2.1. The 2-plane $\mathbf{E}+\alpha$ does not meet $\mathbf{B}$ if and only if $\alpha$ is regular and in this case the tiling defined by $\mathscr{O}$ and $\alpha$ is coincident with the tiling $T_{\alpha}$ obtained by the strip projection method.

It follows that the set $I r$ of all singular points is $\mathbf{p}^{\perp}(\mathbf{B}), \mathbf{I r}=\mathbf{p}^{\perp}(\mathbf{B})$. We have the following description of Ir. For $I=(i, j) \in M$ let $H_{I}$ be the 2-plane spanned by $e_{i}{ }^{\perp}$ and $e_{j}^{\perp}$. Then $H_{I}+\mathbf{p}^{\perp}\left(\mathbb{Z}^{5}\right)$ is a dense family of parallel 2-planes in $\mathbf{E}^{\perp}$. 
Proposition 2.2 (see [ODK]). The set Ir of all singular points is the union of ten families of parallel planes $H_{I}+\mathbf{p}^{\perp}\left(\mathbb{Z}^{5}\right), I \in M$ :

$$
\mathbf{I r}=\mathbf{p}^{\perp}(\mathbf{B})=\bigcup_{I \in M}\left(H_{I}+\mathbf{p}^{\perp}\left(\mathbb{Z}^{5}\right)\right)
$$

Of course $\mathbf{I r}$ is a dense set in $\mathbf{E}^{\perp}$ but it has measure 0 .

\subsection{Index of Rhombs}

We have $\overline{\mathscr{T}}_{t}=\overline{\mathscr{T}}_{t+1}$, hence we study $\overline{\mathscr{T}}_{t}$ for $-1<t \leq 0$.

Recall that a rhomb is always referred to as a translate of one of ten $P_{i}, I \in M$. Suppose a rhomb $P$ has one vertex in $p\left(\mathbb{Z}^{5}\right)$. Then all the four vertices are in $p\left(\mathbb{Z}^{5}\right)$, hence they each have an index (see Section 1.3). Let $v$ and $v^{\prime}$ be two vertices such that the segment $\left[v, v^{\prime}\right]$ is an edge of $P$. Then the vector $\overline{v v^{\prime}}$ is either one of $e_{0}, e_{1}, e_{2}, e_{3}, e_{4}$ or one of $-e_{0},-e_{1},-e_{2},-e_{3},-e_{4}$. In the first case index $\left(v^{\prime}\right)=$ index $(v)+1$, in the second index $\left(v^{\prime}\right)=\operatorname{index}(v)-1$. Hence the four vertices can take only three values of the index $i, i+1, i+2(\bmod 5)$ for some $i \in\{0,1,2,3,4\}$.

Definition. This number $i$ is called the index of this rhomb.

In case $P=\mathbf{p}\left(\xi+C_{I}\right), \xi \in \mathbb{Z}^{5}, I \in M$, it is easy to see that index $(P)=$ index $(p(\xi))$. The index is important due to the following proposition which is a consequence of Proposition 1.2(b).

Proposition 2.3. Suppose $P, P^{\prime}$ are congruent rhombs, $P=P^{\prime}+v$. Then $v$ belongs to $\mathbf{p}(\Lambda)$ if and only if index $(P)=$ index $\left(P^{\prime}\right)$.

Proposition 2.4. For regular $\alpha \in \overline{\mathbf{E}}_{t}$ the tiles of $T_{\alpha}$ have indices 1 or 2 if $t=0$ and indices $0,1,2$ if $-1<t<0$. (The case $t=0$ has been proved in $[d B 1]$.)

Proof. Suppose $P$ is a tile of $T_{\alpha}$. Then $P=\mathbf{p}\left(\xi+C_{I}\right)$ for some prism $\xi+C_{I}$ meeting $\mathbf{E}+\alpha$, by the cut method. Since $\mathbf{E}+\alpha$ does not meet the parallel boundary of the prism $\xi+C_{I}$, it meets the interior of this prism. Projecting on $\Delta$, we have $\mathbf{p}_{\Delta}(\mathbf{E}+\alpha)=t \delta$ and $\mathbf{p}_{\Delta}\left(C_{I}\right)=[\mathbf{0},-3 \delta]$. Let $\mathbf{p}_{\Delta}(\xi)=m \delta, m \in \mathbb{Z}$. Then $m \delta+[0,-3 \delta]$ contains $t \delta$ as an interior point. It follows that, when $t=0, m$ must be 1 or 2 , and when $-1<t<0$, then $m$ must be 0,1 , or 2 . By Proposition 1.2(a) we have $m=\operatorname{index}(\mathrm{p}(\xi))=\operatorname{index}(P)$.

\subsection{The Cut Method in Four-Dimensional Planes}

We have seen that every tiling $T_{\alpha}$ is the slice of a periodic structure in $\mathbb{R}^{5}$ by a 2-plane. For a fixed $t$ every tiling $T_{\alpha}$ with $\alpha \in \overline{\mathbf{E}}_{t}$ can be obtained by a slice of a periodic structure in the 4-plane $\mathbb{R}_{t}^{4}$ as follows.

The intersection of $\mathbb{R}_{t}^{4}$ with a tile $\xi+C_{I}$ of $\theta$ is

$$
\left[\mathbf{p}(\xi)+P_{I}\right]+\left[\overline{\mathbf{E}}_{t} \cap\left(\mathbf{p}^{\perp}(\xi)+P_{I}^{\perp}\right)\right]
$$




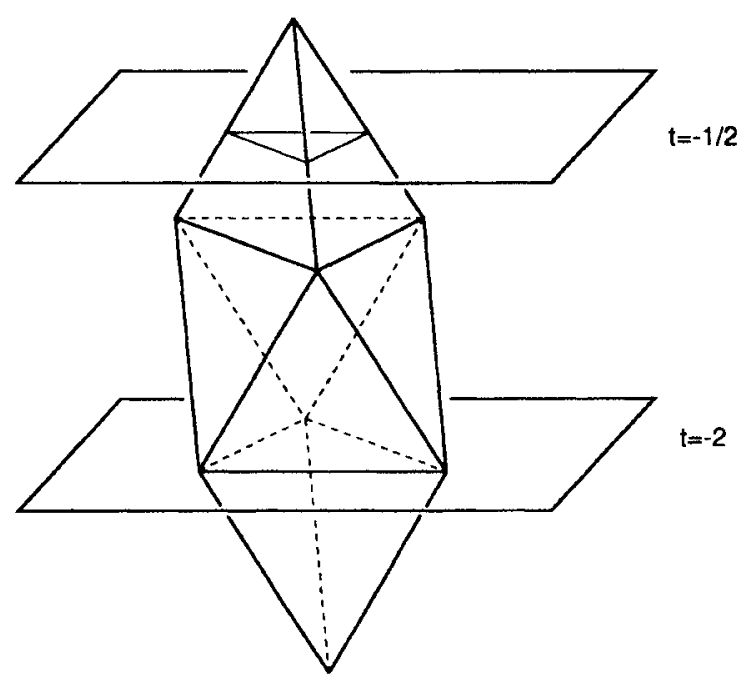

Fig. 3. Intersections of $\overline{\mathbf{E}}_{t}$ and $P_{I}^{\perp}$.

by formula (1). The first term is a translate of the rhomb $P_{I}$, the second term is either a point or a polygon, if it is not empty (see Fig. 3). Hence the intersection of $\mathbb{R}_{t}^{4}$ with a tile of $\mathscr{O}$ is either of dimension 2 or 4 . Let $\mathscr{O}_{t}$ be the family of all the four-dimensional intersections of $\mathbb{R}_{t}^{4}$ with tiles of $\mathscr{Q}$. The family $\mathscr{O}_{t}$ is a tiling of $\mathbb{R}_{t}^{4}$ and has two fundamental properties:

(i) It is invariant under translations from $\Lambda$ (recall that $\Lambda=\mathbb{R}_{0}^{4} \cap \mathbb{Z}^{5}$ is a lattice in $\mathbb{R}_{0}^{4}$ ).

(ii) All its members are prisms; there are a finite number of them up to congruence.

Definition. Any family of four-dimensional polyhedra in $\mathbb{R}_{t}^{4}$ covering $\mathbb{R}_{t}^{4}$ without overlaps and satisfying conditions (i) and (ii) above is called an oblique periodic tiling of $\mathbb{R}_{t}^{4}$.

It follows immediately from the construction that, for a regular $\alpha \in \overline{\mathbf{E}}_{t}$, the tiling $T_{\alpha}$ is obtained by intersecting $\mathbf{E}+\alpha$ with members of the family $\mathscr{O}_{t}$ and then projecting onto $\mathbf{E}$.

Definition. Suppose $C$ is a prism in $\mathbb{R}_{t}^{4}$ such that $\mathbf{p}^{\perp}(C)$ is a polygon. The sum of $\mathbf{p}(C)$ and an edge of polygon $\mathbf{p}^{\perp}(C)$ is called a small wall of $C$. The union of all the small walls of $C$ is its parallel boundary.

Suppose $\mathscr{U}$ is an oblique periodic tiling of $\mathbb{R}_{t}^{4}$ whose tiles are colored polyhedra. Let $\mathbf{B}(\mathscr{U})$ be the union of all the small walls of all the tiles of $\mathscr{U}$. If $\alpha \in \overline{\mathbf{E}}_{t}$ is such that $\mathbf{E}+\alpha$ does not meet $\mathbf{B}(\mathscr{U})$, then by intersecting $\mathbf{E}+\alpha$ with the colored tiles of $\mathscr{U}$ and then projecting onto $\mathbf{E}$ we get a colored tiling, called the colored tiling defined by $\mathscr{U}$ and $\alpha$. Denote by $\mathscr{T}(\mathscr{U})$ the set of all such colored tilings and their 
translates, and by $\overline{\mathfrak{T}} \mathscr{U})$ the closure of $\mathscr{T}(\mathscr{U})$. When $\mathscr{U}$ is a noncolored tiling we use a similar definition, the set $\overline{\mathscr{T}}(\mathscr{U})$ is then a set of noncolored tilings.

Of course $\overline{\mathscr{T}}\left(\mathscr{O}_{t}\right)=\overline{\mathscr{T}}_{t}$. The projection on $\overline{\mathbf{E}}_{t}$ of the parallel boundary $\mathscr{B}(\mathscr{O} t)$ is the set of singular points lying in $\overline{\mathbf{E}}_{t}$. Hence we get

Proposition 2.5. The projection of the parallel boundary $\mathbf{B}\left(\mathscr{O}_{t}\right)$ onto $\overline{\mathbf{E}}_{t}$ is $\operatorname{Ir} \cap \overline{\mathbf{E}}_{t}$ :

$$
\mathbf{p}^{\perp}\left(\mathbf{B}\left(\mathscr{O}_{t}\right)\right)=\mathbf{I r} \cap \overline{\mathbf{E}}_{t}
$$

\subsection{Lifting a Tiling}

Suppose that $\mathscr{U}$ is an oblique periodic tiling of $\mathbb{R}_{t}^{4}$ whose tiles are colored polyhedra, and that $T$ is a colored tiling of $\mathbf{E}$.

Definition. A lift of a tile $P$ of $T$ into $\mathscr{U}$ is a tile $C$ of $\mathscr{U}$ such that $\mathbf{p}(C)=P$ and the colors of $P$ and $C$ are the same. A lift of $T$ into $\mathscr{U}$ is a map $l:\{$ tiles of $T\} \rightarrow$ \{prisms of $\mathscr{U}\}$ such that for every tile $P$ of $T$ the tile $l(P)$ is a lift of $P$ into $\mathscr{U}$.

Of course the lift does not always exist, and when one does exist it may not be unique.

Definition. A lift $l$ of a tiling $T$ into $\mathscr{U}$ is strongly connected if, for every pair of tiles $P_{1}, P_{2}$ sharing a common edge, the polygons $\mathbf{p}^{\perp}\left(l\left(P_{1}\right)\right), \mathbf{p}^{\perp}\left(l\left(P_{2}\right)\right)$ have a common interior point.

A simple but important example is the case when $T$ is a colored tiling defined by $\alpha$ and $\mathscr{U}$, where $\mathbf{E}+\alpha$ does not meet the parallel boundary $\mathbf{B}(\mathscr{U})$. Then $T$ has a lift into $\mathscr{U}: l(P)$, where $P$ is a tile of $T$, is the tile of $\mathscr{U}$ which meets $\mathbf{E}+\alpha$ and projects into $P$. This lift is strongly connected because all the polygons $\mathbf{p}^{\perp}(l(P))$ contain $\alpha$ as an interior point.

Let $P$ be a rhomb having vertices in $\mathbf{p}\left(\mathbb{Z}^{5}\right)$. There may be no lift of $P$ into $\mathscr{O}_{t}$ but there are always lifts of $P$ into $\mathscr{O}$; they are of the form $C+k 5 \delta, k \in \mathbb{Z}$, where $C$ is congruent to one of ten prisms $C_{I}$. The projection $\mathbf{p}_{\Delta}\left(C_{I}\right)$ is the segment $[0,-3 \delta]$. Hence there is at most one prism from the set $C+k 5 \delta$ which meets $\mathbb{R}_{t}^{4}$. Thus we have:

Proposition 2.6. Suppose $P$ is a rhomb having vertices in $\mathrm{p}\left(\mathbb{Z}^{5}\right)$. There is at most one tile $C$ of $\mathscr{Q}_{t}$ such that $\mathrm{p}(C)=P$.

This means the lift into $\theta_{t}$ is always unique. 


\subsection{Singular Cases}

For completeness we describe the "singular tilings," i.e., all the tilings in $\overline{\mathscr{T}} \backslash \mathscr{T}$, $\overline{\mathscr{T}_{t}} \backslash \mathscr{T}_{t}$. The results here are not used in what follows.

Suppose $\alpha \in \mathbf{I r}$. Recall that Ir is a family of 2-planes in $\mathbf{E}^{\perp}$. There are several 2-planes from Ir going through $\alpha$; they partition $\mathbf{E}^{\perp}$ into many pairs, called "corners."

Proposition 2.7. Suppose $\alpha_{i}, i=1,2, \ldots$ are regular, lie in one corner of $\mathbf{E}^{\perp}$ separated by 2-planes from Ir going through $\alpha$, and converge to $\alpha$ when $i \rightarrow \infty$. Then the sequence of tilings $T_{\alpha_{i}}$ converges to a tiling, called the tiling defined by $\alpha$ and this corner. This tiling depends only on the corner containing $\alpha_{i}$, but not on the choice of points $\alpha_{i}$.

Proof. We have to prove that for $r>0$ a number $N$ exists such that if $i>N$, then all the tilings $T_{\alpha_{i}}$ coincide inside the disk $U_{r}$ with center at 0 and radius $r$. Consider the union $Z$ of parallel boundaries of all prisms (from the family $\mathscr{O}$ ) which projects onto $\mathbf{E}$ into rhombs intersecting $U_{r}$. Let $Y=\mathbf{p}^{\perp}(Z)$. Of course $Y \subset \mathbf{I r}$, but it is not a dense set in $\mathbf{E}^{\perp}$ : for large $r$ it divides a small neighborhood of $\alpha$ just as the 2-planes from Ir going through $\alpha$ do. Hence for large $i$ all the points $\alpha_{i}$ lie in one part of $\mathbf{E}$ divided by $Y$. Obviously for these $\alpha_{i}$ the tilings $T_{\alpha_{1}}$ are the same inside $U_{r}$.

It can be proved that no two tilings defined by an irregular $\alpha$ and two different corners are the same, and that the closure $\overline{\mathscr{T}}$ of $\mathscr{T}$ is the set of:

(a) All $T_{\alpha}$ with regular $\alpha$, and their translates.

(b) All tilings defined by singular $\alpha$ and a corner of $\mathbf{E}^{\perp}$ divided by 2-planes from Ir going through $\alpha$, and their translates.

The singular cases in $\overline{\mathscr{T}}_{t}$ are similar. Suppose $\alpha \in \overline{\mathbf{E}}_{t}$ is singular. The set $\operatorname{Ir} \cap \overline{\mathbf{E}}_{t}$ is a family of lines in $\overline{\mathbf{E}}_{t}$. There are several lines in $\operatorname{Ir} \cap \overline{\mathbf{E}}_{t}$ going through $\alpha$; they partition $\overline{\mathbf{E}}_{t}$ into many parts. If $\alpha_{i}$ is a sequence of regular points lying in one part and converging to $\alpha$, then the corresponding tilings converging to a tiling, called the tiling defined by $\alpha$ and this part. Of course this tiling belongs to $\overline{\mathscr{F}}_{t}$ and it can be proved that $\overline{\mathscr{F}}_{t}$, up to translations, is just the set of all such tilings plus "regular" ones. Using this picture the topology of $\overline{\mathscr{T}}, \overline{\mathscr{T}}_{t}$ can be easily described.

\section{The Penrose Tilings}

Theorem 3.1. The set $\overline{\mathscr{T}}_{0}$ admits a local rule of type 2 .

This theorem was first proved by de Bruijn [dB1]. The proof here is different from that of de Bruijn and will be soon generalized for other cases.

We present a concrete local rule which is actually equivalent to the Penrose local rule. We make use of the Main Technical Theorem that is proven in Section 6. 


\subsection{Coloring and the Local Rule for $\overline{\mathscr{T}}_{0}$}

We describe in more detail the tiles (or members) of the family $\mathscr{O}_{0}$. Each tile is an intersection $\left(C_{I}+\xi\right) \cap \mathbb{R}_{0}^{4}$.

Definition. For a tile $C$ of $\mathscr{O}_{0}$ let index $(C)=\operatorname{index}(\mathbf{p}(C))$.

So far we have defined the index for points in $\mathbf{p}\left(\mathbb{Z}^{5}\right)$, for rhombs with vertices in $\mathbf{p}\left(\mathbb{Z}^{5}\right)$, and for tiles of $\mathscr{O}_{0}$.

Proposition 3.2. Up to translations by vectors from $\Lambda$ the family $\mathscr{O}_{0}$ has 20 different members.

Proof. Fix $I \in M$. The intersections $C_{I}^{1}=\left(C_{I}+\varepsilon_{0}\right) \cap \mathbb{R}_{0}^{4}$ and $C_{I}^{2}=\left(C_{I}+2 \varepsilon_{0}\right)$ $\cap \mathbb{R}_{0}^{4}$ are not congruent: their projections on $\overline{\mathbf{E}}$ are two triangles which are not congruent. We prove that every member of $\mathscr{O}_{0}$ is a translate of one of twenty $C_{I}^{j}$, $j=1,2, I \in M$, translated by a vector in $\Lambda$.

A member of $\mathscr{O}_{0}$ is the intersection $\left(\xi+C_{I}\right) \cap \mathbb{R}_{0}^{4}$, where $\mathbb{R}_{0}^{4}$ meets the interior of $\xi+C_{I}$. Then $\mathbf{p}_{\Delta}(\xi)$ must be $j \delta$ where $j=1$ or 2 (see Proposition 2.4). By Proposition 1.2, $\eta=\xi-j \varepsilon_{0}$ belongs to $\Lambda$. The translation by $\eta$ transforms $C_{I}^{j}$ into the intersection $\left(\xi+C_{I}\right) \cap \mathbb{R}_{0}^{4}$.

The index of a tile $C_{I}^{j}+\xi$ of $\mathscr{O}_{0}$ is $j$. In this section we consider the index of a tile of $\mathscr{O}_{0}$ to be its color. Now $\mathscr{O}_{0}$ is an oblique periodic tiling of $\mathbb{R}_{0}^{4}$ whose tiles are colored prisms. We use the notation $\mathscr{Q}_{0}^{c}$ for this colored oblique periodic tiling. If $\alpha \in \overline{\mathbf{E}}$ is regular, let $T_{\alpha}^{\mathrm{c}}$ be the colored tiling defined by $\alpha$ and $\mathscr{O}_{0}^{\mathrm{c}}$; it has 20 prototiles: $\left(P_{I}, 1\right),\left(P_{I}, 2\right), I \in M$. The color of a tile $P$ of $T_{\alpha}^{\mathrm{c}}$ is its index. The set $\overline{\mathscr{T}}\left(\mathscr{O}_{0}^{\mathrm{c}}\right)$ (see the definition in Section 2.3) is the closure of the set of all $T_{\alpha}^{\mathrm{c}}$. If we ignore the color, then $\overline{\mathscr{T}}\left(\mathscr{O}_{0}^{\mathrm{c}}\right)=\overline{\mathscr{T}}_{0}$.

We define the following local rule of type 2 . Let $\mathscr{B}_{0}$ denote the set of all edge-configurations of tilings from $\overline{\mathscr{T}}\left(\mathscr{O}_{0}^{\mathrm{c}}\right)$. Up to congruence there are only a finite number of elements in $\mathscr{B}_{0}$. Then a colored tiling $T$ satisfies the local rule $\mathscr{B}_{0}$ if and only if every edge-configuration of $T$ is congruent to an edge-configuration of a colored tiling $T_{\alpha}^{\mathbf{c}}, \alpha \in \overline{\mathbf{E}}$. A concrete description of this local rule is given later in Section 3.4.

Theorem 3.3. If $T$ is a colored tiling satisfying the local rule $\mathscr{B}_{0}$, then $T$ belongs to $\overline{\mathscr{T}}\left(Q_{0}^{c}\right)$.

Theorem 3.1 is a consequence of this theorem; we see later that this theorem is equivalent to de Bruijn's theorem. We prove this theorem after some preparation.

Let $P$ be an arbitrary rhomb congruent to one of $P_{I}, I \in M$, and having vertices in $\mathbf{p}\left(\mathbb{Z}^{5}\right)$. The index $(P)$ is defined (see Section 2.2) and equals index $(v)$ of some vertex $v$ of $P$. Then index $(P+x)=\operatorname{index}(v+x)$ for every $x \in \mathbf{p}\left(\mathbb{Z}^{5}\right)$. Hence $x \in \mathrm{p}\left(\mathbb{Z}^{5}\right)$ always exists such that index $(P+x)$ is a given number in $\{0,1,2,3,4\}$. 
Consider a colored tiling $T$ satisfying the local rule $\mathscr{B}_{0}$. Every tile of $T$ has color 1 or 2 . If one vertex of $T$ is in $\mathbf{p}\left(\mathbb{Z}^{5}\right)$, then all the vertices of $T$ are in $\mathbf{p}\left(\mathbb{Z}^{5}\right)$. After a translation we may assume that there is a tile $P$ of $T$ such that its color is the same as its index. From now on fix such $T$.

Proposition 3.4. The tiling $T$ has a strongly connected lift into $\mathscr{O}_{0}^{c}$.

Proof. First we prove that for every tile $Q$ of $T$ the index of $Q$ and the color of $Q$ are the same. This is true for $Q=P$.

Suppose $Q_{1}, Q_{2}$ are two tiles of $T$ sharing a common edge, and index $\left(Q_{1}\right)=$ color of $Q_{1}$. By definition of the local rule, there is a pair of tiles $Q_{1}^{\prime}, Q_{2}^{\prime}$ of a colored tiling $T_{\alpha}^{\mathrm{c}}, \alpha \in \overline{\mathbf{E}}$, such that the colored pair $\left(Q_{1}, Q_{2}\right)$ is congruent to the colored pair $\left(Q_{1}^{\prime}, Q_{2}^{\prime}\right)$, where the colors of $Q_{1}^{\prime}, Q_{2}^{\prime}$ are their indices. Since index $\left(Q_{1}\right)=$ color of $Q_{1}=$ index $\left(Q_{1}^{\prime}\right)$, by Proposition 2.3 the pair $Q_{1}, Q_{2}$ is the translation of $Q_{1}^{\prime}, Q_{2}^{\prime}$, by a vector $v=\mathbf{p}(\xi), \xi \in \Lambda$. Then again by Proposition $2.3 \operatorname{index}\left(Q_{2}\right)=\operatorname{index}\left(Q_{2}^{\prime}\right)$. Hence index $\left(Q_{2}\right)=$ color of $Q_{2}$.

Each of $Q_{1}^{\prime}, Q_{2}^{\prime}$ has lift into $\mathscr{O}_{0}^{\mathrm{c}}$. Let the lift of $Q_{i}^{\prime}$ be $C_{i}^{\prime}, i=1,2$. Then the colored tile $C_{i}^{\prime}+\xi$ of $\mathscr{O}_{0}^{\mathrm{c}}$ is the lift of $Q_{i}$. The lift of a colored tile is unique (Proposition 2.6). The projections of $C_{1}^{\prime}$ and $C_{2}^{\prime}$ on $\bar{E}$ have nonempty interior intersection, because both contain $\alpha$ as the interior point. The lifts of $Q_{1}, Q_{2}$ are translations of the lifts of $Q_{1}^{\prime}, Q_{2}^{\prime}$, hence their projections on $\bar{E}$ also have nonempty intersection. So we can lift every colored tile of $T$ into $\mathscr{O}_{0}^{\mathrm{c}}$ and the lift is strongly connected.

The next step is to prove that if a colored tiling $T$ has a connected lift into $\mathscr{O}_{0}^{\mathrm{c}}$, then $T$ belongs to $\overline{\mathscr{Y}}\left(\mathscr{O}_{0}^{\mathrm{c}}\right)$.

Note that the family $\mathscr{O}_{0}$ of prisms has the following symmetries:

(a) Translations by vectors from $\Lambda$.

(b) Actions of cyclic group $\mathbb{Z}_{5}$.

(c) Symmetry with respect to 0 (this maps $C_{I}^{1}$ to $C_{I}^{2}+3 \varepsilon_{0}-\varepsilon_{2}-\varepsilon_{3}-\varepsilon_{4}$ ).

Up to these symmetries in $\mathscr{O}_{0}$ there are only two tiles: their representatives are $C_{(0,1)}^{1}, C_{(0,2)}^{1}$. This will cut significantly the number of verifications which appear later in the proofs.

\subsection{Properties of $\mathscr{O}_{0}^{c}$}

Consider five 2-planes, $F_{i}$ spanned by $\varepsilon_{i+1}-\varepsilon_{i-1}$ and $\varepsilon_{i+2}-\varepsilon_{i-2}$ for $i=0,1,2,3,4$ (indices are taken mod 5). They are rational 2-planes contained in $\mathbb{R}_{0}^{4}$. An important remark is that the intersection of these 2-planes with $\mathbf{E}$ and $\overline{\mathbf{E}}$ are lines, i.e., they are not in generic position with respect to $\mathbf{E}$ and $\overline{\mathbf{E}}$. Let $f_{i}=F_{i} \cap E, \bar{f}_{i}=F_{i} \cap \bar{E}$. Then both $f_{i}$ and $\bar{f}_{i}$ are lines and $F_{i}=f_{i}+\bar{f}_{i}$. All of the 2-planes $F_{i}$ are prisms and $\mathbf{p}\left(F_{i}\right)=f_{i}, \mathbf{p}^{\perp}\left(F_{i}\right)=\bar{f}_{i}$. 
Let $\mathscr{F}_{i}=F_{i}+\Lambda$, for $i=0,1,2,3,4$, and $\mathscr{F}=\bigcup_{i=0}^{4} F_{i}$. Each $\mathscr{F}_{i}$ is a family of parallel 2-planes in $\mathbb{R}_{0}^{4}$; this family is locally finite (i.e., every compact set meets only a finite number of 2-planes from this family) because $F_{i}$ is a rational 2-plane.

Proposition 3.5. If two 2-planes $F, F^{\prime}$ in $\mathscr{F}$ have the same projection onto $\overline{\mathbf{E}}$, then $F=F^{\prime}$.

Proof. We may assume that $F=F_{i}$ and $F^{\prime}=F_{i}+\xi$ where $\xi \in \Lambda$, for some $i$. Then $\mathbf{p}^{\perp}\left(F_{i}\right)=\mathbf{p}^{\perp}\left(F_{i}+\xi\right)$, or, equivalently, $\xi \in\left(F_{i}+\mathbf{E}\right)$. Note that $F_{i}+\mathbf{E}$ is a homogeneous 3-plane. If $\xi$ does not belong to $F_{i}$, then the 3-plane spanned by $\xi$ and $F_{i}$ must coincide with $F_{i}+\mathbf{E}$. However, the 3-plane spanned by $\xi$ and $F_{i}$ is rational, so it could not contain $\mathbf{E}$ (Proposition 1.2). Hence $\xi \in F_{i}$. This means $F=F^{\prime}$.

For each $i=0,1,2,3,4$ the set $\Phi_{i}=\mathbf{p}^{\perp}\left(\mathscr{F}_{i}\right)=\bar{f}_{i}+\mathbf{p}^{\perp}(\Lambda)$ is a dense family of parallel lines in $\overline{\mathbf{E}}$. Denote by $\Phi$ the union of five sets $\Phi_{i}$. Then $\Phi=\mathbf{p}^{\perp}(\mathscr{F})$.

Proposition 3.6. The projection of the parallel boundary $\mathbf{B}\left(\mathscr{O}_{0}^{c}\right)$ onto $\overline{\mathbf{E}}$ is $\Phi$ :

$$
\mathbf{p}^{\perp}\left(\mathbf{B}\left(\mathscr{G}_{0}^{\mathrm{c}}\right)\right)=\Phi
$$

Proof. By Propositions 2.2 and 2.5, $\mathbf{p}^{\perp}\left(\mathbf{B}\left(\mathscr{G}_{0}^{\mathrm{c}}\right)\right)=\mathbf{p}^{\perp}\left(\mathbf{B}\left(\mathscr{G}_{0}\right)\right)$ is the intersection of $\overline{\mathbf{E}}$ with ten families of planes $H_{I}+\mathbf{p}^{\perp}\left(\mathbb{Z}^{5}\right), I \in M$. These ten families can be grouped into five pairs, each pair has the same intersection with $\overline{\mathbf{E}}$, and the intersections are exactly the five families $\Phi_{i}$ of parallel lines, because $\bar{f}_{i}=H_{(i+1, i-1)} \cap$ $\overline{\mathbf{E}}=H_{(i+2, i-2)} \cap \bar{E}$ (indices are taken $\bmod 5$ ).

We suppose the length of vector $e_{0}$ is 1 . Let $U_{d}$ be the disk in $\mathbf{E}$ of radius $d$ centered at $\mathbf{0}$. The set $F_{i}+U_{d}$ is a three-dimensional prism, its projection onto $\overline{\mathbf{E}}$ is the line $\bar{f}_{i}$ (just as the projection of $F_{i}$ ), and its projection onto $\mathbf{E}$ is $f_{i}+U_{d}$ which is a strip of width $2 d$.

Definition. In this section any set of the type $F_{i}+U_{d_{0}}+\xi$, where $i=0,1,2,3,4, \xi$ $\in \Lambda, d_{0}=(3-\sqrt{5}) / 4$, is called $a$ big wall.

The union $\mathscr{W}_{d_{0}}$ of all the big walls is $\mathscr{F}+U_{d_{0}}$. The next proposition is a specific property of the case $t=0$.

Proposition 3.7. All the big walls are contained in the parallel boundary: $\mathscr{W}_{d_{0}} \subset \mathbf{B}\left(\mathscr{O}_{0}^{\mathrm{c}}\right)$.

Proof. We have to prove that the intersection of every tile of $\mathscr{\sigma}_{0}$ with every big wall is contained in $\mathbf{B}\left(\mathscr{O}_{0}^{\mathrm{c}}\right)$. The intersection is of two types: of dimension 3 or of dimension less than 3 , because the dimension of a wall is 3 .

First we prove that all the intersections of the first type are in $\mathbf{B}\left(\mathscr{O}_{0}^{c}\right)$. Since $\mathbf{B}\left(\mathscr{O}_{0}^{c}\right)$ and $\mathscr{W}_{d_{0}}$ are invariant under the symmetries listed in (a)-(c) in Section 3.1, it suffices to prove that the intersection of dimension 3 of the prism $C_{I}^{1}$ with a big wall lies on 
the parallel boundary of the prism $C_{I}^{1}$ where $I=(0,1)$ or $(0,2)$. In fact it is easy to check that, for each $I=(0,1)$ or $(0,2)$, the prism $C_{I}^{1}$ intersects at most three big walls if the intersection is of dimension 3 , and in this case the intersection lies on the parallel boundary of $C_{I}^{1}$. Hence the intersection of the first type of every big wall with every tile of $\mathscr{\theta}_{0}$ is in $\mathbf{B}\left(\mathscr{O}_{0}^{\mathrm{c}}\right)$.

Now let $W$ be a big wall. Since $W$ and $\mathbf{B}\left(\mathscr{O}_{0}^{\mathrm{c}}\right)$ are closed subsets of $\mathbb{R}_{0}^{4}$, the complement $W \backslash \mathbf{B}\left(\mathscr{O}_{0}^{\mathrm{c}}\right)$, as a subset of $W$, is open. By the above result, this complement is the union of at most a countable number of sets of dimension less than 3 and hence must be empty. That is, $W \subset \mathbf{B}\left(\mathscr{O}_{0}^{\mathrm{c}}\right)$.

We recall that a small wall of a prism $C$ is the sum of $\mathbf{p}(C)$ and one edge of $\mathbf{p}^{\perp}(C)$. The projection of a small wall of a tile of $\mathscr{G}_{0}^{c}$ on $\overline{\mathbf{E}}$ is a segment lying in $\Phi=\mathbf{p}^{\perp}(\mathscr{F})$, hence it lies in the projection of a big wall. Proposition 3.5 says that this big wall is unique.

Proposition 3.8. If the projection of a small wall $w$ on $\overline{\mathbf{E}}$ lies in the projection of a big wall $W$, then the small wall has nonempty intersection with the big wall.

Proof. It suffices to consider the cases when $w$ is a small wall of one of the $C_{l}^{l}$, where $I=(0,1)$ or $(0,2)$. In each case $C$ has three small walls and the assertion can be verified straightforwardly by finding the unique big wall involved and checking that the projections of the small walls and the big wall onto $\mathbf{E}$ have nonempty intersection.

\subsection{Proof of Theorem 3.3 Using the Main Technical Theorem}

The following theorem is proved in Section 6.

Theorem 3.9 (Main Technical Theorem). Let $\mathscr{U}$ be an oblique periodic colored tiling of $\mathbb{R}_{0}^{4}$ satisfying the following conditions for some constant $d>0$ :

(a) The projection of the parallel boundary onto $\overline{\mathbf{E}}$ is $\Phi: \mathbf{p}^{\perp}(\mathbf{B}(\mathscr{U}))=\Phi$.

(b) $\mathscr{W}_{d}=\mathscr{F}+U_{d}$ is a subset of the parallel boundary.

(c) If $w$ is a small wall of a tile of $\mathscr{U}$ and the segment $\mathbf{p}^{\perp}(w)$ lies on the line $\mathbf{p}^{\perp}(F)$, where $F$ is a 2-plane in $F$, then $w$ and $F+U_{d}$ have nonempty intersection.

Then if $T$ is a colored tiling having a strongly connected lift into $\mathscr{Z}, T$ belongs to $\overline{\mathscr{T}}(\mathscr{U})$.

By Propositions 3.6-3.8, the oblique periodic colored tiling $\mathscr{O}_{0}^{\mathrm{c}}$ satisfies conditions (a)-(c) of the Main Technical Theorem. By this theorem if $T$ has a connected lift, then $T$ belongs to $\overline{\mathscr{T}}\left(\mathscr{O}_{0}^{\mathrm{c}}\right)$. Theorem 3.3 is proved. 

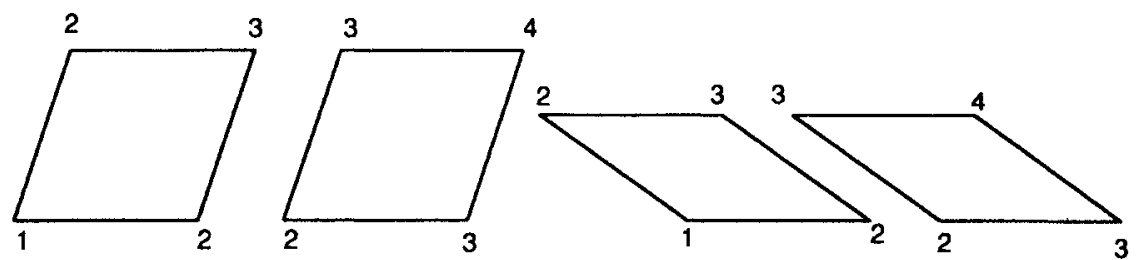

Fig. 4. Prototiles of $\overline{\mathscr{T}}\left(\mathscr{O}_{0}^{\mathrm{c}}\right)$.

\subsection{Connection to Penrose Local Rule}

First we describe the local rule $\mathscr{B}_{0}$. The prototiles of this local rule are $\left(P_{1}, j\right)$, $I \in M, j=1,2$. We have to point out all the possible edge-configurations in $\overline{\mathscr{T}}\left(\mathscr{O}_{0}^{\mathrm{c}}\right)$. For every $P j=\mathbf{p}(C j)$ we mark the vertices of polygon $P j$ by their indices. Then we have 20 rhombs with marked vertices. Four of them are as in Fig. 4 (in fact two of them are congruent to $P_{(0,1)}$, two are congruent to $\left.P_{(0,2)}\right)$. All the other rhombs are obtained from these four by rotations by $2 m \pi / 5, m \in \mathbb{Z}$.

There is one-to-one corresponding between the 20 prototiles $\left(P_{I}, j\right), I \in M$, $j=1,2$, and these 20 marked rhombs. We describe the local rule $\mathscr{B}_{0}$ in terms of the 20 marked rhombs.

Proposition 3.10. The local rule $\mathscr{B}_{0}$ is the set of edge-configurations:

(a) Shown in Fig. 5 together with their reflections with respect to a horizontal line.

(b) Obtained by rotating any edge-configurations in (a) by $2 m \pi / 5, m \in \mathbb{Z}$.

This can be verified by analyzing the edge-configurations of $T_{\alpha}^{\mathrm{c}}$.

The local rule $\mathscr{B}_{0}$ is equivalent to the Penrose local rule in the sense that every colored tiling satisfying this local rule can be equipped with arrows such that the arrowed tiling satisfies the Penrose local rule, and, conversely, every tiling satisfying the Penrose local rule can be colored into a colored tiling satisfying the local rule $\mathscr{B}_{0}$. This can be seen as follows.

For every marked rhomb we put a double arrow on every edge with vertices 2 and 1 such that the arrow directs to 1 . We also put a double arrow on every edge with vertices 3 and 4 such that the arrow directs to 4 . Then every rhomb of the 20 rhombs has double arrows on two or four its edges. On the other two edges we put single arrows such that they direct to obtuse angles of the rhomb. By this way we get the 20 arrowed rhombs of Penrose. Then it can be checked easily that an edge-configuration belongs to the local rule $\mathscr{B}_{0}$ if and only if arrows on the common edge coming from different rhombs are the same. Conversely, every tiling satisfying the Penrose local rule can be marked (indexed) at vertices such that (when forgetting about arrows) this tiling satisfies the local rule $\mathscr{B}_{0}$ (see [dB1]). Hence the local rule constructed here and the Penrose local rule are equivalent. 

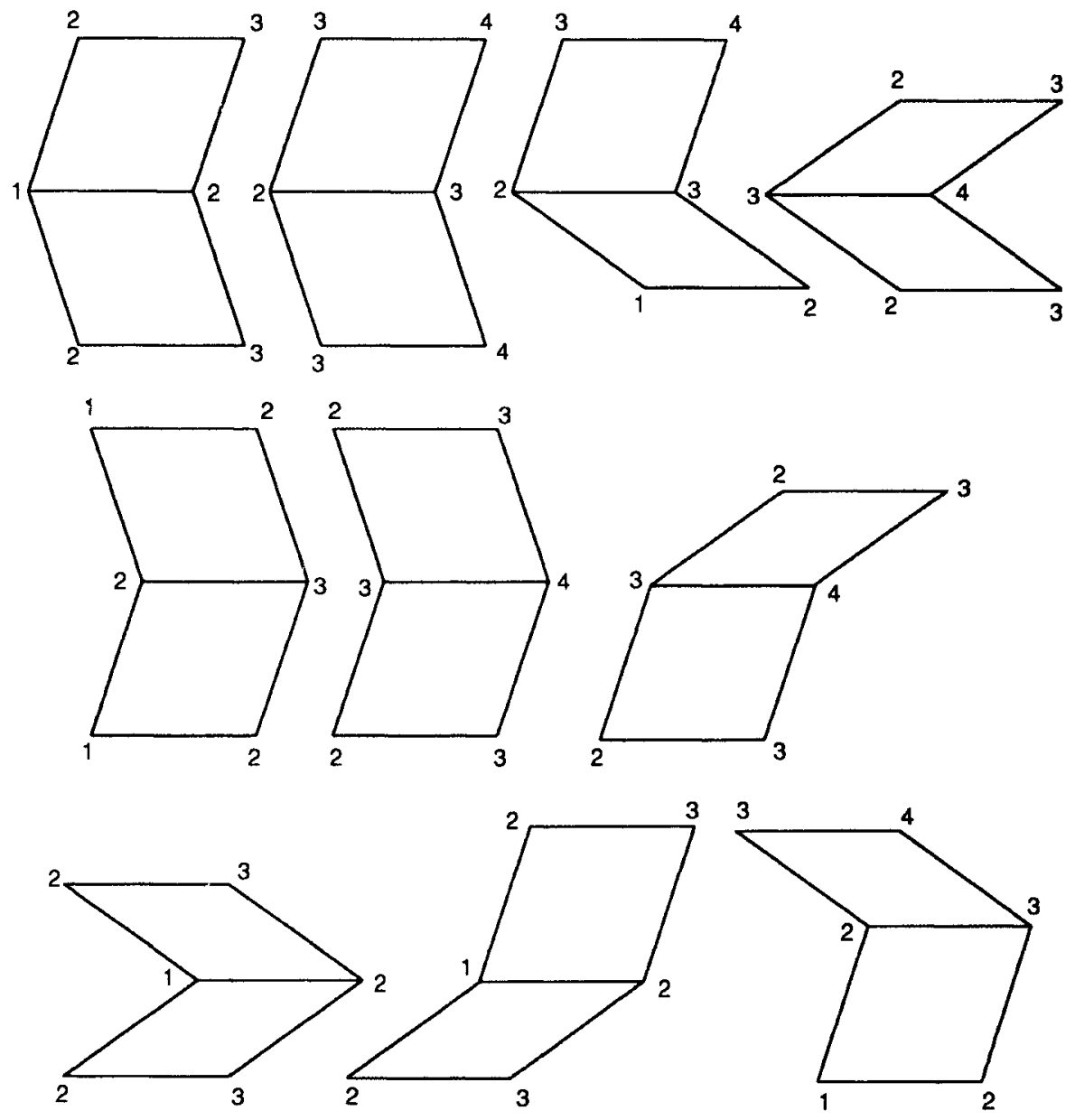

Fig. 5. The local rule for $\ddot{\mathscr{T}}\left(\mathscr{C}_{0}^{\mathrm{c}}\right)$.

\section{A Local Rule of Type 2 for $t=(m+n \sqrt{5}) / q$}

Theorem 4.1. If $t=(m+n \tau) / q$, where $m, n, q$ are integers and $\tau=(1+\sqrt{5}) / 2$, then the set $\overline{\mathscr{F}}_{t}$ admits a local rule of type 2.

The proof is in fact simpler than the previous case. The reason is that in the previous case we wanted to establish not only the existence of a local rule but also the coincidence with the Penrose local rule. Here we may simplify the proof but the local rules we obtain are much more complicated. In the previous sections we had to prove that all the big walls are contained in the parallel boundary and if a small wall has projection onto $\overline{\mathbf{E}}$ lying in the projection of a big wall, then the small wall and the big wall have nonempty intersection. Here these properties are fulfilled automat- 
ically from the construction of the refined oblique periodic tiling. An example of the local rule for the case $t=\frac{1}{2}$ is presented in Section 7 .

\subsection{The Big Walls}

First we describe the tiles of $\mathscr{O}_{t}$. Let

$$
C_{I, t}^{j}=\left(C_{I}+j \varepsilon_{0}\right) \cap \mathbb{R}_{t}^{4}
$$

For $-1<t<0$ none of the three sets $C_{Y, t}^{j}, j=0,1,2$, is empty, and no two of them are congruent. Using the same proof of Proposition 3.2 it can be proved that every tile of $\mathscr{O}_{t}$ is the translation of one of 30 prisms $C_{I, t}^{j}$ by a vector in $\Lambda$ :

$$
\mathscr{G}_{t}=\left\{C_{I, t}^{j}+\xi, I \in M, j=0,1,2, \xi \in \Lambda\right\} \text {. }
$$

Definition. For a tile $D$ of $\mathscr{O}_{t}$ let $\operatorname{index}(D)=\operatorname{index}(\mathrm{p}(D))$.

Then the index of a tile $C_{I, t}^{j}+\xi$ of $\mathscr{O}_{t}$, where $\xi \in \Lambda$, is $j$.

By Propositions 2.2 and 2.5 the projection of the parallel boundary $\mathbf{B}\left(\mathscr{Q}_{t}\right)$ on $\overline{\mathbf{E}}_{t}$ is the union of ten families of lines $\left[H_{I} \cap \overline{\mathbf{E}}_{t}\right]+\Lambda$. When $t=(m+n \tau) / q$ all the ten families of lines are subsets of a system of lines which is as "good" as $\Phi$ (this is the place where we use $t=(m+n \tau) / q))$ :

Proposition 4.2. If $t=(m+n \tau) / q$, then the projection of $\mathbf{B}\left(\mathscr{O}_{t}\right)$ onto $\overline{\mathbf{E}}_{t}$, after translation by $-t e_{0}^{\perp}$, is contained in $\Phi / q$, where $\Phi / q=\{x \mid q x \in \Phi\}$ :

$$
\mathbf{p}^{\perp}\left(\mathbf{B}\left(\mathscr{O}_{t}\right)\right) \subset\left(t e_{0}^{\perp}+\Phi / q\right) \text {. }
$$

Proof. Using Propositions 2.2 and 2.5, it suffices to verify that each line $H_{I} \cap \overline{\mathbf{E}}_{t}$ is lying in the set $t e_{0}^{\perp}+\Phi / q$. This can be done straightforwardly.

Let $\hat{\mathscr{F}_{i}}=F_{i}+\Lambda / q+t e_{0}^{\perp}$, and $\hat{\mathscr{F}}=\bigcup_{i=0}^{4} \hat{\mathscr{F}_{i}}$. Each $\hat{\mathscr{F}_{i}}$ is a locally finite family of parallel 2-planes in $\mathbb{R}_{t}^{4}$. We have $\mathbf{p}^{\perp}(\hat{\mathscr{F}})=\Phi / q+t e_{0}^{\perp}$.

Proposition 4.3. If $\mathbf{p}^{\perp}(F)=\mathbf{p}^{\perp}\left(F^{\prime}\right)$ where $F, F^{\prime}$ are 2-planes from $\hat{\mathscr{F}}$, then $F=F^{\prime}$.

Proof. After translation by $-t e_{0}^{\perp}$ and rescaling by $q$ from the system of 2-planes $\hat{\mathscr{F}}$ we get the system $\mathscr{F}$. Hence this proposition follows from Proposition 3.4.

The projection of a small wall of a tile of $\theta_{t}$ onto $\overline{\mathbf{E}}_{t}$ is a segment lying in the projection of some 2-plane in $\hat{\mathscr{F}}$ onto $\overline{\mathbf{E}}_{t}$. Up to translations by vectors in $\Lambda$ there are only a finite number of small walls of the tiles of $\mathscr{O}_{t}$. Hence there is a constant $d>0$ such that if $w$ is a small wall of a tile of $\theta_{t}$ and the segment $\mathbf{p}^{\perp}(w)$ is contained in the line $\mathrm{p}^{\perp}(F)$, where $F$ is a 2 -plane in $\hat{F}$, then the Hausdorff distance between the 
line $\mathbf{p}(F)$ and the rhomb $\mathbf{p}(w)$ is less than $d$. In other words, the sets $\mathbf{p}(F)+U_{d}$ and $\mathbf{p}(w)$ must have a common point, where $U_{d}$ is the disk in $\mathbf{E}$ with center at $\mathbf{0}$ and radius $d$. We fix such a $d$.

Definition. In this section a set $F+U_{d}$ where $F$ is a 2 -plane in $\hat{F}$ is called a big wall.

\subsection{The Refinement of $\mathscr{O}_{t}$}

Each big wall $F+U_{d}$ is contained in a unique 3-plane $F+\mathbf{E}$. We construct an oblique periodic tiling of $\mathbb{R}_{t}^{4}$ such that all the big walls are contained in the parallel boundary of this olique periodic tiling.

Every compact set in $\mathbb{R}_{t}^{4}$ meets only a finite number of 2-planes $\hat{\mathscr{F}}$ and hence meets only a finite number of big walls. Each prism $C_{I, t}^{j}$ meets a finite number of big walls. The 3-planes going through these big walls divide $C_{I, t}^{j}$ into many pieces. Each piece is also a prism, and the projection of each piece onto $\mathbf{E}$ is the same as the projection of $C_{I, t}^{j}$ onto E. Suppose there are $s$ pieces; we denote them by $C_{I, t}^{j ; 1}, C_{i, t}^{j ; 2}, \ldots, C_{I, t}^{j ; s}$, where $s$ depends on $I$ and $j, s=s(I, j)$.

So far we have divided every $C_{l, t}^{j}, I \in M, j=0,1,2$, into smaller prisms. Every tile of $\mathscr{Q}_{t}$ is a translate of one of $C_{I, t}^{j}, I \in M, j=0,1,2$, and we repeat the division in every tile of $\mathscr{O}_{t}$, by translation. We get a new family of (smaller) prisms, each of the form $C_{I, t}^{j ; k}+\xi$ where $I \in M, j \in\{0,1,2\}, k \in\{1, \ldots, s(I, j)\}, \xi \in \Lambda$. We define the color of the prism $C_{l, t}^{j ; k}+\xi$ as the pair $(j ; k)$. Denote this new family of colored prisms by $\hat{\mathscr{Q}}_{t}^{c}$. It is an oblique periodic colored tiling of $\mathbb{R}_{t}^{4}$. The color of a tile contains the index of this tile. From the construction we have:

Proposition 4.4. The parallel boundary $\mathrm{B}\left(\hat{\mathscr{O}}_{t}^{\mathrm{c}}\right)$ contains all the big walls and has the same projection on $\overline{\mathbf{E}}_{t}$ as the big walls:

$$
\mathbf{p}^{\perp}\left(\mathbf{B}\left(\hat{\mathscr{O}}_{t}^{\mathrm{c}}\right)\right)=\mathbf{p}^{\perp}(\hat{\mathscr{F}})=\Phi / q+t e_{0}^{\perp}
$$

Proposition 4.5. Every colored rhomb in $\mathbf{E}$ has at most one lift onto $\hat{\mathscr{O}}_{t}^{\mathrm{c}}$.

Proof. This follows from Proposition 2.6 and the coloring.

If $w$ is a small wall of a tile $C$ of $\hat{\mathscr{O}}_{t}^{\mathrm{c}}$, then $\mathbf{p}^{\perp}(w)$ is a segment lying on some line $\mathbf{p}^{\perp}(W)$, where $W$ is a big wall.

Proposition 4.6. If the projection of a small wall $w$ of a prism $C$ of $\hat{\mathscr{O}}_{t}^{\mathrm{c}}$ onto $\overline{\mathbf{E}}_{t}$ lies on the line $\mathbf{p}^{\perp}(W)$, where $W$ is a big wall, then $w$ and $W$ have nonempty intersection.

Proof. $C$ is contained in a prism $D$ of the original family $\mathscr{O}_{t}$ with $\mathbf{p}(C)=\mathbf{p}(D)$. Let $Q=\mathbf{p}^{\perp}(D)$. Then $\mathbf{p}^{\perp}(C)$ is a polygon lying in $Q$, and $\mathbf{p}^{\perp}(w)$ is an edge of $\mathbf{p}^{\perp}(C)$. 
There are two possibilities:

(a) $\mathbf{p}^{\perp}(w)$ does not lie in any edge of $Q$. Then by the construction of the family $\hat{\mathcal{G}}_{t}^{\mathrm{c}}$ there must be a big wall meeting $D$ and projecting into the line containing $\mathbf{p}^{\perp}(w)$. This big wall must be $W$ because it has the same projection on $\overline{\mathbf{E}}_{t}$ as $W$ has. Hence $W$ and $D$ have nonempty intersection, so $\mathrm{p}(W)$ and $\mathbf{p}(D)=$ $\mathrm{p}(w)$ have nonempty intersection. Then $w$ and $W$ also have nonempty intersection because they are prisms and their projections both on $\mathbf{E}$ and on $\overline{\mathbf{E}}$ have nonempty intersection.

(b) $\mathbf{p}^{\perp}(w)$ is a segment lying in a side of $Q$. By definition of $d, \mathbf{p}(W)$ and $\mathbf{p}(D)=\mathbf{p}(w)$ have nonempty intersection. We can apply the last phrase of the previous paragraph.

\subsection{Proof of Theorem 4.1}

The oblique periodic colored tiling $\hat{\mathscr{O}}_{t}^{\mathrm{c}}$ defines a set of colored tilings $\overline{\mathscr{T}}\left(\hat{\mathscr{O}}_{t}^{\mathrm{c}}\right)$. If $\alpha$ does not belong to $\Phi / q+t e_{0}^{\perp}=\mathbf{p}^{\perp}\left(\mathbf{B}\left(\hat{\mathscr{O}}_{t}^{\mathrm{c}}\right)\right)$, then $\alpha$ and $\hat{\mathscr{\theta}}_{t}^{\mathrm{c}}$ define a colored tiling $T_{\alpha}^{\mathrm{c}}$ which is the same as $T_{\alpha}$ if we ignore the colors.

We define $\mathscr{B}_{t}$ as the set of all edge-configurations of all colored tilings in $\overline{\mathscr{T}} \hat{\mathscr{O}}_{t}^{\mathrm{c}}$ ). Up to translation there are only a finite number of edge-configurations in $\mathscr{B}_{t}$. By definition, $\mathscr{B}_{t}$ is a local rule of type 2 .

Theorem 4.7. Every colored tiling satisfying the local rule $\mathscr{B}_{t}$ is a tiling of $\overline{\mathscr{T}}\left(\hat{\mathscr{O}}_{t}^{\mathrm{c}}\right)$.

Theorem 4.1 is a consequence of this theorem. Again we use the Main Technical Theorem to prove Theorem 4.7.

Suppose $T$ is colored tiling satisfying the local rule $\mathscr{B}_{t}$. Recall that a lift of $T$ into $\hat{\theta}_{t}^{\mathrm{c}}$ is a map $l$ : $\{$ tiles of $T\} \rightarrow$ \{prisms of $\left.\hat{\mathscr{O}}_{t}^{\mathrm{c}}\right\}$ such that $\mathrm{p}(l(P))=P$ and the color of $P$ is the color of $l(P)$ for every tile $P$ of $T$.

Proposition 4.8. If $T$ is a tiling satisfying the local rule $\mathscr{B}_{t}$, then, after a translation, $T$ has a strongly connected lift onto $\hat{\mathscr{\theta}}_{t}^{\mathrm{c}}$.

Proof. Using the uniqueness of the lift of a colored tile (Proposition 4.5), the proof is the same as in the case $t=0$ (see Proposition 3.4).

The oblique periodic tiling $\hat{\theta}_{t}^{c}$ does not satisfy the conditions of the Main Technical Theorem, because it is not a tiling of $\mathbb{R}_{0}^{4}$. However, after translation by $-t e_{0}^{\perp}$ and rescaling by $q$ from $\hat{\mathcal{O}}_{t}^{\mathrm{c}}$ we get an oblique periodic colored tiling of $\mathbb{R}_{0}^{4}$ satisfying all the conditions of the Main Technical Theorem, by Propositions 4.4 and 4.6. Hence we can use this theorem to conclude that $T$ belongs to $\overline{\mathscr{T}}\left(\hat{\mathscr{O}}_{t}^{c}\right)$. Theorem 4.7 is proved. 


\section{Local Rules of Type 1 for $t=m+n \tau$}

At the outset $\overline{\mathscr{T}}_{t}$ is a set of tilings without any colors. Hence the question of finding local rules of type 1 (which does not involve any coloring) for these sets is the most natural.

Theorem 5.1. If $t=m+n \tau$, where $m, n$ are integers and $\tau=(1+\sqrt{5}) / 2$, then the set $\overline{\mathscr{F}}_{t}$ admits a local nule of type 1 .

It follows from a result of Ingersent and Steinhardt [IS] that if $t \neq m+n \tau$, where $\tau=(1+\sqrt{5}) / 2$, then the set $\overline{\mathscr{T}}$ does not admit any local rule by type 1 (see Proposition 5.9 below). Hence $t=m+n \tau$ is a criterion for the existence of a local rule of type 1 .

The case $t=0$ is well known [dB2], [Se], and it suffices to take the radius of the local rule $r=2$. The proof consists of the following observation. If $T$ is a tiling in $\overline{\mathscr{T}}_{0}$, then by inspecting the neighborhood within radius 2 of any tile we can decide how to put arrows on edges of this tile such that the arrowed tiling is a Penrose arrowed tiling. The proof for the case $t=m+n \tau$ is based on the same idea.

Suppose $t=m+n \tau$ is fixed, $-1<t \leq 0$. Let $\mathscr{A}(r)$ be the set of all $r$-maps of all the tilings of $\overline{\mathscr{T}_{t}}$. Up to translations there are only a finite number of elements in the set $\mathscr{N}(r)$. This set $\mathscr{A}(r)$ is a local rule of type 1 . We prove that, for sufficiently large $r$, the set of all tilings satisfying the local rule $\mathscr{A}(r)$ is coincident with $\overline{\mathscr{T}}_{t}$. In the previous section we defined the oblique periodic colored tiling $\hat{\mathscr{C}}_{t}^{\mathrm{c}}$. When $t=m+n \tau$, by Proposition 4.4 , the projection of the parallel boundary $\mathbf{B}\left(\hat{\mathscr{O}}_{t}^{\mathrm{c}}\right)$ onto $\hat{E}_{t}$ is the same as the projection of the parallel boundary $\mathbf{B}\left(\mathscr{O}_{t}\right)$ of the original oblique periodic tiling $\mathscr{O}_{t}$,

$$
\mathbf{p}^{\perp}\left(\mathbf{B}\left(\hat{\mathscr{O}}_{t}^{\mathrm{c}}\right)\right)=\mathbf{p}^{\perp}\left(\mathbf{B}\left(\mathscr{\sigma}_{t}\right)\right)=\Phi+t e_{0}^{\perp}=\mathbf{I r} \cap \overline{\mathbf{E}}_{t}
$$

If $\alpha \in \overline{\mathbf{E}}_{t}$ is regular we denote, as in the previous section, the colored tiling defined by $\alpha$ and $\mathscr{\mathscr { O }}_{t}^{\mathrm{c}}$ by $T_{\alpha}^{\mathrm{c}}$.

\section{1. r-Maps and Coloring}

Fix $t=m+n \tau$. For a rhomb $P$ with vertices in $\mathbf{p}\left(\mathbb{Z}^{5}\right)$, index $(P)$ is the index of a unique vertex of $P$, called the base vertex of $P$. For a regular $\alpha \in \overline{\mathbf{E}}_{t}$ we have the colored tiling $T_{\alpha}^{\mathrm{c}}$ and the noncolored underlying tiling $T_{\alpha}$.

Proposition 5.2. There is $r_{0}>0$ such that, for every two congruent tiles $P, P^{\prime}$ of $T_{\alpha}$, if the $r_{0}$-maps at the base vertices of these tiles are congruent, then the colors of $P$ and $P^{\prime}$ in $T_{\alpha}^{\mathrm{c}}$ are the same. 
First we show how to deduce Theorem 5.1 from this proposition. Fix a tiling $T$ satisfying $\mathscr{A}(r)$ with $r>r_{0}+2$. A priori there are no colors on tiles of $T$. We color them as follows.

Let $P$ be a tile of $T$ with base vertex $v$. The $r_{0}$-map of $T$ at $v$ is congruent to an $r_{0}$-map of $T_{\alpha}$; let $Q$ be the tile of $T_{\alpha}$ corresponding to $P$ under this congruence. We color $P$ by the color of $Q$ in $T_{\alpha}^{\mathrm{c}}$. This way we color all the tiles of $T$.

If $P, P^{\prime}$ are tiles of $T$ sharing a common edge, then the colored edge configuration $\left(P, P^{\prime}\right)$ is congruent to a colored edge-configuration of $T_{\alpha}^{\mathrm{c}}$, since $r>r_{0}+2$. By Theorem 4.7 the colored tiling $T$ belongs to $\overline{\mathscr{T}}\left(\hat{Q}_{t}^{\mathrm{c}}\right)$. Hence the noncolored tiling $T$ belongs to $\overline{\mathscr{T}}_{t}$.

It remains to prove Proposition 5.2. The idea is as follows. We define $r$-volumes which correspond to vertices with the same $r$-maps, and the volume of colored tiles which corresponds to tiles of the same colors of $T_{\alpha}^{\mathrm{c}}$. For large $r$ we prove that every $r$-volume cannot meet two different volumes of colored tiles.

\section{2. r-Volumes}

First we recall the concept of $r$-volumes. In this subsection $\alpha$ is not necessarily in $\overline{\mathbf{E}}_{t}$.

For a point $x \in \mathbf{p}^{\perp}\left(\mathbb{Z}^{5}\right)$ there is a unique $\xi \in \mathbb{Z}^{5}$ such that $\mathbf{p}^{\perp}(\xi)=x$. Define $\varphi(x)=\mathbf{p}(\xi)$. The map $\varphi$ is defined only for points in $\mathbf{p}^{\perp}\left(\mathbb{Z}^{5}\right) \subset U_{k \in \mathbb{Z}} \overline{\mathbf{E}}_{k}$ and is not one-to-one. If $\varphi(x)=v$, then $\varphi^{-1}(v)=\{x+k 5 \delta, k \in \mathbb{Z}\}$. For a set $X$ in $\mathbf{E}^{1}$ we define $\tilde{\varphi}(X)$ as the set $\varphi\left(X \cap \mathbf{p}^{\perp}\left(\mathbb{Z}^{5}\right)\right)$.

Let $K=\mathbf{p}^{\perp}(\gamma)=\operatorname{Pol}\left(e_{0}^{\perp}, \ldots, e_{4}^{\perp}\right)$; it is a polyhedron in $\mathbf{E}^{\perp}$ with 22 vertices, 40 edges, and 20 facets (Fig. 6). The projection of $K$ on $\Delta$ is the segment $[0,5 \delta]$. For

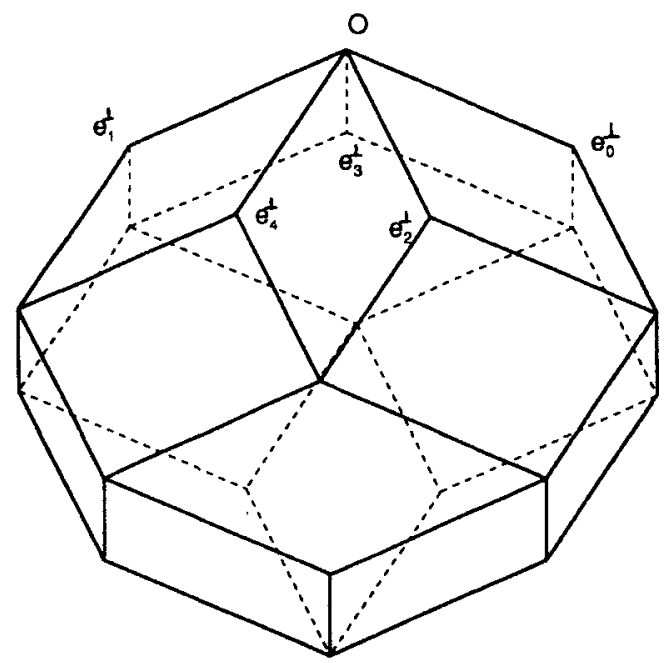

Fig. 6. The polyhedron $K$. 
every $x \in \mathbf{p}\left(\mathbb{Z}^{5}\right)$, the set $K-\varphi^{-1}(x)$ is the union of infinitely many translates of $K$, any two of them differ by a multiple of $5 \delta$, hence at most two of them meets $K$.

For a regular $\alpha \in \mathbf{E}^{\perp}$ the set of vertices of $T_{\alpha}$ is $\tilde{\varphi}(\alpha+K)$. The following proposition follows easily.

Proposition 5.3. Two points $x, x+v$ are vertices of a tiling $T_{\alpha}$ if and only if $x$ belongs to $\tilde{\varphi}\left\{\alpha+\left[K \cap\left(K-\varphi^{-1}(v)\right)\right]\right\}$.

Now fix $r>0$. Let $\mathscr{R}$ be an $r$-map at some vertex $x$. If $x+v$ is a vertex of $\mathscr{R}$, then there is path connecting $x$ and $x+v$ by edges of $\mathscr{R}$. Each edge is a translate of $e_{i}, i=0, \ldots, 4$, hence using the path we can write $v=\sum_{i=0}^{4} n_{i} e_{i}, n_{i} \in \mathbb{Z}$. This presentation of $v$ as an integer linear combination of the $e_{i}$ 's does not depend on the path in $\mathscr{R}$ connecting $x$ and $x+v$. Let $\psi(v)=\sum_{i=0}^{4} n_{i} e_{i}^{\perp}$ and $K_{\mathscr{R}}=K \cap$ $\left(\cap_{\nu} K-\psi(v)\right)$, where $v$ runs the set of all vertices of $\mathscr{R}$. Note that $\psi(v)$ is a point in $\varphi^{-1}(v)$. The set $K_{\mathscr{R}}$ is called the volume of $\mathscr{R}$. An r-volume is the volume of an $r$-map. It follows from the previous proposition:

Proposition 5.4. The r-map of $T_{\alpha}$ at $v$ is congruent to $\mathscr{R}$ if and only if $v$ belongs to $\tilde{\varphi}\left(\alpha+K_{\mathscr{R}}\right)$.

There are only a finte number of $r$-maps; and the polyhedron $K$ is partitioned into $r$-volumes, each corresponds to an $r$-map. The partition can be described as follows. Let $\sigma(r)$ be the set of all vectors in $\mathbf{p}\left(\mathbb{Z}^{5}\right)$ whose length is less than $r$. All the polyhedra $K-\varphi^{-1}(v)$, when $v \in \sigma(r)$ together divide $K$ into $r$-volumes. Note that there are only a finite number of points in $\sigma(r)$ such that $K-\varphi^{-1}(v)$ meets $K$. The significance of the $r$-volumes is expressed in the following:

Proposition 5.5. For a regular $\alpha \in \mathbf{E}^{\perp}$, two r-maps of $T_{\alpha}$ at vertices $v, v^{\prime}$ are congruent if and only if there is an r-volume $Y$ such that both $v, v^{\prime}$ belong to $\tilde{\varphi}(\alpha+Y)$.

This follows from Proposition 5.4.

The polyhedron $K$ has 20 facets, each a translate of one of ten parallelograms $\operatorname{Pol}\left(e_{i}^{\perp}, e_{j}^{\perp}\right),(i, j) \in M$, by vectors in $\mathbf{p}^{\perp}\left(\mathbb{Z}^{5}\right)$. If we use translations $n_{1} e_{i}^{-}+n_{2} e_{j}^{\perp}$, $n_{1}, n_{2} \in \mathbb{Z}$, then the translates of $\operatorname{Pol}\left(e_{i}^{\perp}, e_{j}^{\perp}\right)$ cover the whole plane $H_{(i, j)}$ spanned by $e_{i}^{\perp}, e_{j}^{\perp}$. Moreover, every compact set in $H_{(i, j)}$ can be covered by a finite number of such translates of $\operatorname{Pol}\left(e_{i}^{\perp}, e_{j}^{\perp}\right)$. Recall that $\mathbf{I r}=\mathrm{U}_{I \in M}\left(H_{I}+\mathbf{p}^{\perp}\left(\mathbb{Z}^{5}\right)\right)$ (see Proposition 2.2).

Proposition 5.6. If $X$ is a polyhedron lying in $K$ and having all facets lying in Ir, then, for sufficiently large $r$, every $r$-volume is either contained in $X$ or does not meet the interior of $X$.

Proof. By the observation preceding the proposition, there are a finite number of points $y_{1}, \ldots, y_{q} \in \mathbf{p}^{\perp}\left(\mathbb{Z}^{5}\right)$ such that all the facets of $X$ lie in the union of all the facets of the polyhedra $K, K-y_{1}, \ldots, K-y_{q}$. Let $r>\max _{j=1, \ldots, q}\left|\varphi\left(y_{j}\right)\right|$. Then by construction every $r$-map is either contained in $X$ or does not have interior intersection with $X$. 
Proposition 5.7. There is an $r_{1}$ such that, for every regular $\alpha \in \mathbf{E}^{\perp}$, the $r_{1}$-maps of $T_{\alpha}$ at vertices of different indices are not congruent.

Proof. Using the previous proposition we see that when $r \rightarrow \infty$, the maximal diameter of $r$-volumes tends to zero. Hence there is $r_{1}$ such that the projection of every $r_{1}$-volume on $\Delta$ is a segment having length less than the length of $[0, \delta]$. The $r_{1}$-maps of vertices of different indices in $T_{\alpha}$ are different, because if two integer points having different index, then they project on $\Delta$ into two points whose distance is greater or equal to the distance between 0 and $\delta$.

Remark. In fact one can take $r_{1}=3$.

Proposition 5.8. If $X$ is a polygon lying in a 2-plane $\overrightarrow{\mathbf{E}}_{u}, u \in \mathbb{R}$, and having edges lying in $\mathbf{I r} \cap \overline{\mathbf{E}}_{u}$, then for sufficiently large $r$ the intersection of every $r$-volume with $\overline{\mathbf{E}}_{u}$ is either contained in $X$ or does not meet the interior of $X$.

Proof. This is a straight corollary of Proposition 5.6.

Proposition 5.9 [IS]. If $\overline{\mathscr{F}_{t}}$ admits local rules of type 1 , then $t$ is of the form $m+n \tau$ for some integers $m, n$.

Proof. The $r$-maps which apear in $T_{\alpha}, \alpha \in \overline{\mathbf{E}}_{\ell}$, are those which correspond to the $r$-volumes which meets the 2-planes $k \delta-\overline{\mathbf{E}}_{t}, k \in \mathbb{Z}$. Suppose $t$ is not of the form $m+n \tau$. Fix $r>0$. The facets of every $r$-volume are in Ir, hence every vertex of it is the intersection point of three different 2-planes in Ir. It is easy to check that the projection of every intersection point of three 2-planes in Ir onto $\Delta$ is of the form $(m+n \tau) \delta$ (see the description of $\mathbf{I r}$ in Proposition 2.2). Hence the planes $k \delta-\overline{\mathbf{E}}_{t}$, $k \in \mathbb{Z}$, can never meet vertices of any $r$-maps. It follows that, for $t^{\prime}$ very close to $t$, the plane $k \delta-\overline{\mathbf{E}}_{t^{\prime}}$ meets the same $r$-volumes as $k \delta-\overline{\mathbf{E}}_{t}$ does. This means every tiling in $\overline{\mathscr{T}_{t}}$, has the same $r$-maps as tilings in $\overline{\mathscr{T}_{t}}$.

\subsection{Proof of Proposition 5.2}

We fix $t=m+n \tau$. Recall that the prototiles of $\hat{\mathscr{O}}_{t}^{\mathrm{c}}$ are $C_{i, t}^{j ; k}$, where $I \in M$, $j=0,1,2, k=1, \ldots, s(I, j)$ (see Section 4.2). In what follows we drop the subscript t. Let $Q_{i}^{j ; k}=\mathbf{p}^{\perp}\left(C_{j}^{j ; k}\right)$ and $P_{i}^{j k}=\mathbf{p}\left(C_{i}^{j ; k}\right)$. Then $P P^{j k}=P_{I}+j e_{0}$ is a rhomb congruent to $P_{I}$ and has base vertex $j e_{0}$; we color $P^{j ; k}$ by $(j, k)$. It follows from formula (2) that $\tilde{Q}_{I}^{j ; k}=j e_{0}^{\perp}-Q_{I}^{j ; k}$ is a polygon lying in the intersection of $K$ with $j \delta-\overline{\mathbf{E}}_{t}$. The following proposition describes $\tilde{Q}_{I}^{j ; k}$ as the "volume" of $P ; ; k$.

Proposition 5.10. Let $\alpha \in \overline{\mathbf{E}}_{t}$ be regular. A vertex $v$ of $T_{\alpha}^{\mathrm{c}}$ is a base vertex of a colored tile congruent to $P j ; k$ if and only if

$$
v \in \tilde{\varphi}\left(\alpha+\tilde{Q}_{I}^{j ; k}\right)
$$


Proof.

(4)

$$
\begin{array}{lll}
\Leftrightarrow & v-j e_{0} \in \tilde{\varphi}\left(\alpha-Q_{I}^{j ; k}\right) & \left.\quad \text { (since } \varphi\left(e_{0}^{\perp}\right)=e_{0}\right) \\
\Leftrightarrow & \exists \xi \in \Lambda \mid \mathbf{p}(\xi)=v-j e_{0} & \text { and } \alpha+\mathbf{E}-Q_{I}^{j ; k} \text { contains } \xi \\
& \quad \text { (since } \alpha-Q_{I}^{j ; k} \subset \mathbb{R}_{0}^{4} \text { ) } \\
\Leftrightarrow \quad \exists \xi \in \Lambda \mid \mathbf{p}(\xi)=v-j e_{0} & \text { and } \alpha+\mathbf{E} \text { meets } \xi+C_{I}^{j ; k} \\
\Leftrightarrow & \exists \xi \in \Lambda \mid \mathbf{p}(\xi)=v-j e_{0} \quad \text { and } \mathbf{p}\left(\xi+C_{Y}^{j, k}\right) \text { is a colored tile of } T_{\alpha}^{\mathrm{c}} .
\end{array}
$$

However, $\mathbf{p}\left(\xi+C_{Y}^{j, k}\right)$ is the colored tile congruent to $P_{I}^{j, k}$ with base point $v$.

Now we can prove Proposition 5.2. Every edge of $Q_{I}^{j, k}$ lies in $\mathbf{p}^{\perp}\left(\mathbf{B}\left(\hat{\mathscr{O}}_{t}^{\mathrm{c}}\right)\right.$, hence every edge of $\tilde{Q}_{I}^{j, k}$ lies in Ir $\cap\left(j \delta-\overline{\mathbf{E}}_{t}\right)$, by (3). By Proposition 5.8 there is $r_{0}$ such that for every polygon $\tilde{Q}_{I}^{j, k}$ the intersection of every $r_{0}$-volume with every plane $k \delta-\overline{\mathbf{E}}_{t}, k \in \mathbb{Z}$, either lies in this polygon or does not have interior intersection with it.

Now suppose $P, P^{\prime}$ are two congruent tiles of $T_{\alpha}$ with base vertices $v, v^{\prime}$, respectively. By Proposition 5.4 both $v, v^{\prime}$ belong to $\tilde{\varphi}(\alpha+Y)$ for some $r_{0}$-volume $Y$. Let the color of $P$ be $(j, k)$. Then $v$ belongs to $\tilde{\varphi}\left(\alpha+\tilde{Q}_{I}^{j, k}\right)$ by Proposition 5.10. Hence $Y \subset \tilde{Q}_{I}^{j, k}$. This, in turn, implies $v^{\prime} \in \tilde{\varphi}\left(\alpha+\tilde{Q}_{Y}^{j, k}\right)$. By Proposition 5.10 the tile $P^{\prime}$ has color $(j, k)$, the same as $P$.

Together with Proposition 2 we have proved Theorem 5.1.

\section{The Main Technical Theorem}

Now we prove the Main Technical Theorem (Theorem 3.8). We reduce the problem to investigating a set of oriented lines on a plane. Similar proofs have appeared in [LPS1] and [LPS2].

Definition. An oriented line on a plane is a line equipped with an open half-plane divided by this line, called the positive half-plane of the oriented line. A set $X$ of points is greater than an oriented line if all points of $X$ are in the positive half-plane. A set of oriented lines is compatible if there is a point greater than all of them. A set of oriented lines is weakly compatible if every finite subset is compatible.

In order to prove the Main Technical Theorem we have to prove that every strongly connected list into $\mathscr{U}$ has to define a tiling in $\mathscr{T} \mathscr{U})$. We need to prove that there is a plane parallel to $\mathbf{E}$ meeting all the members of the lift (actually with some extra conditions for the singular case). This is equivalent to the fact that the projections of all the members of the lift onto $\overline{\mathrm{E}}$ have a common point. Note that the projection of evey tile of $\mathscr{O}_{t}$ onto $\overline{\mathbf{E}}$ is a polygon. If we orient all the lines going through an edge of the projection of a member of the lift so that the interior of the projection is greater than this line, then it is enough to prove that there is a point 
greater than any line or at least lying on this line. We prove an even stronger statement: these lines are weakly compatible.

In what follows we introduce orientations for lines of an even larger set (it is $\Phi$ ), since it is easier to deal with this larger set. We then prove that this set of oriented lines is weakly compatible, using the "bootstrapped" property and the compatibility of every three lines meeting at a point.

\subsection{Plan of the Proof of the Main Technical Theorem}

Fix a tiling $T$ having a strongly connected lift into $\mathscr{U}$. First we prove:

Proposition 6.1. For every finite set of tiles $P_{1}, \ldots, P_{m}$ of $T$, the projections $\mathbf{p}^{\perp}\left(l\left(P_{1}\right)\right), \ldots, \mathbf{p}^{\perp}\left(l\left(P_{m}\right)\right)$ have nonempty interior intersection.

Then we use the following:

Proposition 6.2. Suppose that the lift $l$ of a colored tiling $T$ satisfies Propositon 6.1. Then $T$ is a colored tiling belonging to $\overline{\mathscr{X}}(\mathscr{U})$.

Theorem 3.8 followed from these propositions. For a regular $\alpha \in \overline{\mathbf{E}}$ the colored tiling defined by $\alpha$ and $\mathscr{U}$ is denoted by $T(\alpha, \mathscr{U})$.

Proof of Proposition 6.2. We number the tiles of $T, T=\left\{P_{1}, P_{2}, \ldots\right\}$ so that for every $r$ the disk $U_{r}$ is covered by the first $N$ tiles, here $N$ depends on $r$. Since the polygons $\mathbf{p}^{\perp}\left(l\left(P_{1}\right)\right), \ldots, \mathbf{p}^{\perp}\left(l\left(P_{N}\right)\right)$ have nonempty interior intersection, the intersection of these polygons is a polygon (with nonempty interior). There is a regular point $\alpha_{r} \in \overline{\mathbf{E}}$ belonging to this polygon. Then the colored tiling $T\left(\alpha_{r}, \mathscr{U}\right)$ is the same as $T$ inside the disk $U_{r}$. Of course $\alpha_{r} \rightarrow \alpha$ when $r \rightarrow \infty$ and $T\left(\alpha_{r}, \mathscr{U}\right) \rightarrow T$ when $r \rightarrow \infty$.

It remains to prove Proposition 6.1. Note that every edge of a polygon $\mathbf{p}^{\perp}$ $(l(P)), P \in T$, is lying on a line in $\Phi$. To prove Proposition 6.1 we introduce orientations on all the lines in $\Phi$ such that:

(*) If the polygon $\mathbf{p}^{\perp}(l(P))$, where $P$ is a tile of $T$, has an edge lying on a line $h$, then the interior of this polygon is greater than $h$.

(**) The set $\Phi$ of oriented lines is weakly compatible.

Proposition 6.1 follows immediately from $(*)$ and $(* *)$.

Our task is to find, for every connected lift $l$ of a colored tiling $T$, a way to orient all the lines in $\Phi$ satisfying conditions $(*)$ and $(* *)$.

Definition. In this section a set $F+U_{d}$ where $F$ is a 2-plane in $F$ is called a big wall. 


\subsection{Orientations of Lines}

We define a multivalued map $\rho: \mathbf{E} \rightarrow \overline{\mathbf{E}}$ as follow. For a point $x$ which is an interior puint of a tile $P$ of $T$ let $\rho(x)$ be the interior of the polygon $\mathbf{p}^{\perp}(l(P))$. Suppose $x$ is not an interior point of any tile, let $P_{1}, \ldots, P_{k}$ be the tiles of $T$ containing $x$ and let $y_{1}, \ldots, y_{k}$ be, respectively, interior points of $P_{1}, \ldots, P_{k}$. Define $\rho(x)=\bigcup_{i=1}^{k} \rho\left(y_{i}\right)$.

If $x$ is an interior point of a tile of $T$, then the set $x+\rho(x)$ does not meet the parallel boundary $\mathbf{B}(\mathscr{U})$. For a set $X$ in $\mathbf{E}$ we define $\rho(X)$ as the union of all $\rho(x)$ where $x \in X$. The following is a consequence of the strong connectedness of the lift.

Proposition 6.3. For every path-connected set $X$ in $\mathbf{E}$ the set $\rho(X)$ is connected.

Suppose $W$ is a big wall: $W=F_{i}+\xi+U_{d}, \xi \in \Lambda$. Then it is a prism, its projection on $\overline{\mathbf{E}}$ is a line contained in $\Phi$, and its projection on $\mathbf{E}$ is a strip, $\mathbf{p}(W)=f_{i}+\mathbf{p}(\xi)+U_{d}$.

Lemma 6.4. If $W$ is a big wall, then $\rho(\mathbf{p}(W))$ does not meet the line $\mathbf{p}^{\perp}(W)$.

Proof. Suppose $y$ is a point lying in both $\rho(\mathbf{p}(W))$ and $\mathbf{p}^{\perp}(W)$. Then $y \in \rho(x)$ for $x \in \mathbf{p}(W)$. Since $d>0$ we can choose $x$ such that it in anterior point of a tile of $T$. Then we see that $x+\rho(x)$ contains $x+y$ which belongs to $W$ because $W$ is a prism. This means that set $x+\rho(x)$ meets $W$, but by condition (c) all the big walls are contained in $\mathbf{B}_{0}$, and hence does not meet $x+\rho(x)$. The proposition follows.

Now we can introduce orientations for the lines in $\Phi$. For every line $h$ in $\Phi$ there is a unique big wall $W$ which projects into $h, \mathbf{p}^{\perp}(W)=h$. By the previous lemma, the set $\rho(\mathbf{p}(W))$ does not meet the line $h$. Since $\rho(\mathbf{p}(W))$ is a connected set, it lies in one half-plane of $\overline{\mathbf{E}}$ separated by $h$. We define the orientation of $h$ such that $\rho(\mathbf{p}(W))$ is greater than $h$.

Proposition 6.5. Suppose $\mathbf{p}^{\perp}(F), \mathbf{p}^{\perp}\left(F^{\prime}\right)$, and $\mathbf{p}^{\perp}\left(F^{\prime \prime}\right)$, where $F, F^{\prime}$, and $F^{\prime \prime}$ are 2-planes in $\mathscr{F}$, have a common point. Then $F, F^{\prime}, F^{\prime \prime}$ also have a common point.

Proof. It is easy to see that no two of $F, F^{\prime}, F^{\prime \prime}$ are parallel. Let $v_{1}, v_{2}, v_{3}$ be three intersection-points of three pairs from $F, F^{\prime}, F^{\prime \prime}$. Then they have rational coordinates and have the same projection on $\overline{\mathbf{E}}$, hence they must be coincident by Proposition 1.2(c).

\subsection{Property (*)}

Suppose $C=l(P)$ is a lift of a tile of $T$. Then $\mathbf{p}^{\perp}(C)$ is a polygon having edges lying in $\Phi$.

Proposition 6.6. Suppose $Q=\mathbf{p}^{\perp}(C)$ has an edge $s$ lying on a line $f$ in $\dot{\Phi}$. Then the interior of $Q$ is greater than $f$. 
Proof. There is a big wall $W$ such that $f=\mathbf{p}^{\perp}(W)$. The set $\mathbf{p}(C)+s$ is a small wall. By condition (c) of the Main Technical Theorem the small wall $p(C)+s$ and the big wall $W$ have a common point $y$. Let $x=\mathbf{p}(y)$. Then $\rho(x)$ contains the interior of $\mathbf{p}^{\perp}(C)$ and by definition of the orientation $\rho(x)>f$, hence the interior of $Q$ is greater than $f$.

\subsection{Property (**)}

The following is crucial.

Proposition 6.7. If several lines in $\Phi$ have a common point, then they are compatible.

Proof. Suppose $\mathbf{p}^{\perp}(F), \mathbf{p}^{\perp}\left(F^{\prime}\right), \ldots, \mathbf{p}^{\perp}\left(F^{\prime \prime}\right)$ have a common point where $F, F^{\prime}, \ldots, F^{\prime \prime}$ are 2-planes from $\mathscr{F}$, then by Proposition $6.5 F, F^{\prime}, \ldots, F^{\prime \prime}$ also have a common point $y$. Let $x=\mathrm{p}(y)$. By definition the set $\rho(x)$ is greater than all the lines $\mathbf{p}^{\perp}(F), \mathbf{p}^{\perp}\left(F^{\prime}\right), \ldots, \mathbf{p}^{\perp}\left(F^{\prime \prime}\right)$.

Proposition 6.8. The set $\rho(\mathbf{E})$ is bounded in $\mathbf{E}$.

The boundedness of $\rho(\mathbf{E})$ is an expression of the "weak local rule" in the sense of Levitov [Lev]. The proof is presented in the Appendix.

Three sets $X, Y, Z$ are called bootstrapped if $X \cap Y=X \cap Z=Y \cap Z$.

Proposition 6.9. Every three of five sets $\mathscr{F}_{0}, \mathscr{F}_{1}, \mathscr{F}_{2}, \mathscr{F}_{3}, \mathscr{F}_{4}$ are bootstrapped. In other words, through every intersection point of 2-planes from two of three families $\mathscr{F}_{i}, \mathscr{F}_{j}, \mathscr{F}_{k}$ passes a 2-plane from the third family.

Proof. Every $\mathscr{F}_{i}$ contains $\Lambda$. It suffices to prove that if $0 \leq i<j \leq 4$, then $\mathscr{F}_{i} \cap \mathscr{F}_{j}$ $=\Lambda$. We assume that $i=0$ and $j=1$ (the other cases are similar). Consider two 2-planes $F_{0}+\xi_{0}$ and $F_{1}+\xi_{1}$ where $\xi_{0}, \xi_{1} \in \Lambda$. Note that $F_{0}$ is spanned by $v_{1}=\varepsilon_{4}-\varepsilon_{1}$ and $v_{2}=\varepsilon_{3}-\varepsilon_{2}$, and $F_{1}$ is spanned by $v_{3}=\varepsilon_{0}-\varepsilon_{2}, v_{4}=\varepsilon_{4}-\varepsilon_{3}$. These four vectors $v_{1}, v_{2}, v_{3}, v_{4}$ generate $\Lambda$ as a $\mathbb{Z}$-module, so we have $\xi_{0}=\sum_{i=1}^{4} a_{i} v_{i}$, $\xi_{1}=\sum_{i=1}^{4} b_{i} v_{i}$ where $a_{i}, b_{i} \in \mathbb{Z}$. Then the point $\left(a_{3} v_{3}+a_{4} v_{4}\right)+\left(b_{1} v_{1}+b_{2} v_{2}\right)$ obviously belongs to both $F_{0}$ and $F_{1}$ and it also belongs to $\Lambda$.

Definition. Two parallel oriented lines have the same orientation if the intersection of their positive half-planes is a half-plane.

The property of the same direction is an equivalent relation between oriented parallel lines. 
The family $\Phi$ of oriented lines satisfies the following:

(i) If several lines from $\Phi$ intersect at a point, then they are compatible.

(ii) Every three of five sets $\Phi_{i}$ are bootstrapped.

(iii) Each family of parallel lines $\Phi_{i}$ are not of the same direction.

(i) is Proposition 6.7, (ii) follows from Propositions 6.9 and 6.6, and (iii) follows from Proposition 6.8 .

Proposition 6.10. There is a point $\alpha \in \overline{\mathbf{E}}$ such that for every line $f$ in $\Phi$ either $\alpha \in f$ or $\alpha$ is greater than $f$.

Proof. We divide the proof into three steps.

Step 1. For every pair of lines in $\Phi_{i}=\mathbf{p}^{\perp}\left(\mathscr{F}_{i}\right)$ there is a point greater than both of them.

Proof. We may assume $i=0$. We suppose the converse, then there are two lines $f, f^{\prime}$ in $\Phi_{0}$ as shown in Fig 7, where the positive half-plane is shadowed. A line $h$ in $\Phi_{1}$ intersects $f, f^{\prime}$ at $x_{0}$ and $y_{0}$ as in Fig. 7. By (ii) there are lines in $\Phi_{2}$ going through $x_{0}$ and $y_{0}$, and by applying this again and again, we get a system of lines as in Fig. 8 where two horizontal lines are $f$ and $f^{\prime}$, all the others are lines in $\Phi_{1}$ or $\Phi_{2}$, and all intersection-points are triple. There are two possible cases of orientation of $h$ as indicated in Fig. 9(a) and (b).

Consider the case in Fig. 9(a). By (i) there is a point greater than all the lines going through $x_{0}$. Hence the orientation of the line $x_{0} y_{0}$ can be found easily. Then applying (i) to points $x_{1}, y_{2}, x_{2}$, etc., we see that all the lines $x_{1} y_{1}, x_{2} y_{2}, x_{3} y_{3}$, etc., have the same orientation (Fig. 10). This contradicts (iii).

In the case in Fig. 9(b) all the lines $x_{0} y_{0}, x_{-1} y_{-1}, x_{-2} y_{-2}$, etc., have the same orientation, which is also a contradiction.

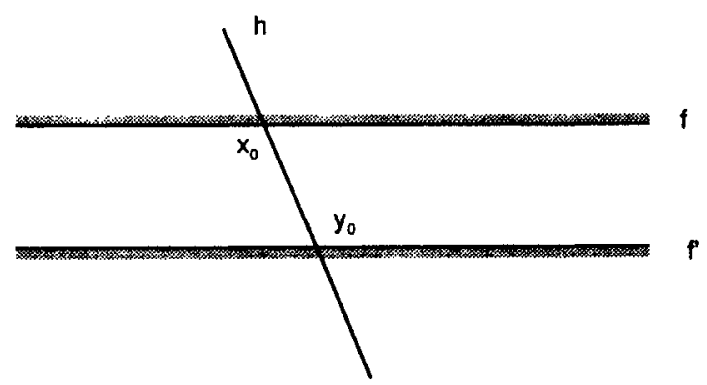

Fig. 7. Two noncompatible parallel lines. 


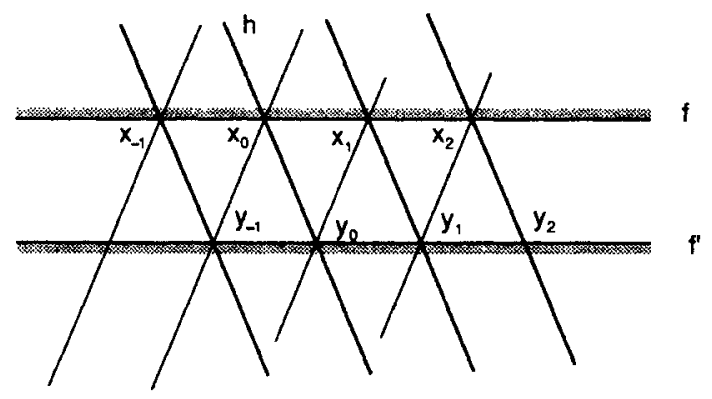

Fig. 8. System of bootstrapping lines.

Step 2. A line $h_{i}$ parallel to all the lines in $\Phi_{i}$ exists such that for every line $f$ in $\Phi_{i}$ either $h_{i}=f$ or $h_{i}$ is greater than $f$.

Proof. This trivially follows from the previous step and the fact that all the lines in $\Phi_{i}$ cannot have the same orientation.

Let $\alpha$ be the intersection point of $h_{0}$ and $h_{1}$.

Step 3. For every line $f$ in $\Phi$ either $\alpha \in f$ or $\alpha$ is greater than $f$.

Proof. Suppose $\alpha$ does not lie in $f$. Let $x_{0}, x_{1}$ denote the intersection-points of $f$ and, respectively, $h_{0}$ and $h_{1}$. Because the set of projections (on $\overline{\mathbf{E}}$ ) of points from $\Lambda$ is dense in $f$ there is a point $y \in f$ lying between $x_{0}$ and $x_{1}$ and $y$ is the projection of a point from $\Lambda$.

There are lines from $\Phi_{0}$ and $\Phi_{1}$ going through $y$ (Fig. 11), and $\alpha$ is greater than them. By using (i) to point $y$ we see that $\alpha>f$.

Now we can prove property $(* *)$.

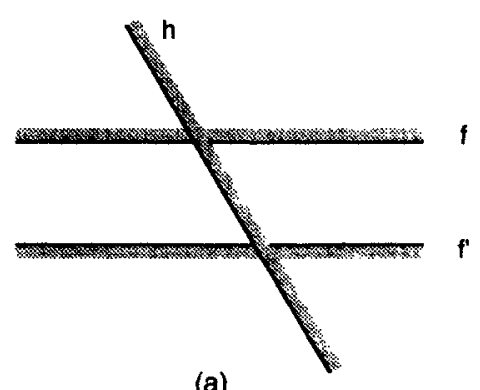

(a)

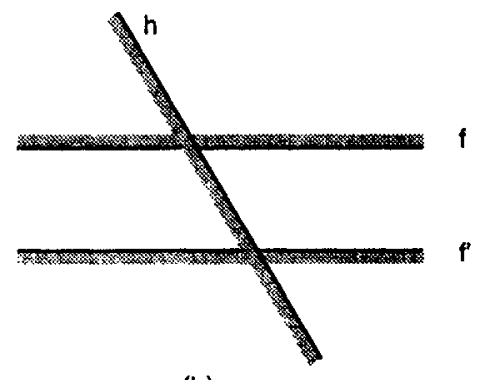

(b)

Fig. 9. Two cases in the proof of Step 1. 


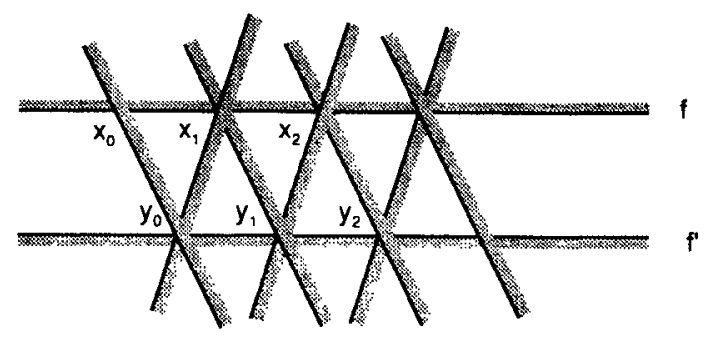

Fig. 10. Orientation of bootstrapping line.

Proposition 6.11. For every finite number of lines $g_{1}, g_{2}, \ldots, g_{m}$ in $\Phi$ there is a point greater than all of them.

Proof. If the point $\alpha$ defined above does not belong to any of $g_{i}, i=1, \ldots, m$, then the point $\alpha$ of the previous proposition is a point to find. Suppose some of $g_{i}$ contains $\alpha$, say $g_{1}, g_{2}, \ldots, g_{n}$, where $n \leq m$. Since $\alpha$ is greater than $g_{i}$ for $i=n+1, \ldots, m$ there is a neighborhood $V$ of $\alpha$ such that $V>g_{i}$ for $i=n+$ $1, \ldots, m$. Since all the lines $g_{i}$ for $i=1, \ldots, n$ go through $\alpha$, there is a point greater than all of them by Proposition 6.7. The set of all points greater than all $g_{i}$ for $i=1, \ldots, n$ is a corner (or an angle) with vertex at $\alpha$ and this set has nonempty intersection with $V$. Hence any point in this intersection is greater than all $g_{i}$, for $i=1, \ldots, m$.

Property $(* *)$ is established, and Theorem 3.8 is proved.

\section{Concluding Remarks, Example: $t=\frac{1}{2}$}

\subsection{On Local Rules of Type 2}

We have a criterion for the existence of local rules of type 1. As for the local rules of type 2 we have proved only the sufficient condition for the existence: $t=$

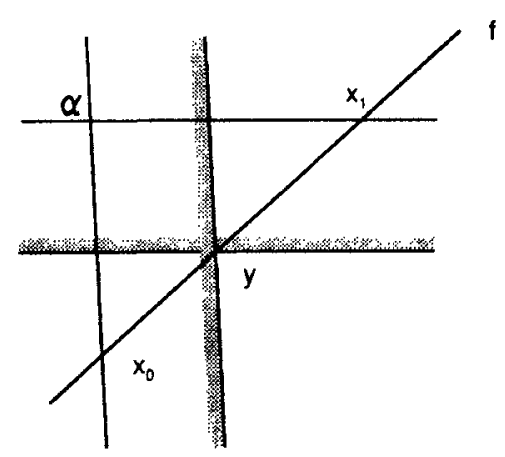

Fig. 11. $\alpha$ is greater than $f$. 
$(m+n \sqrt{5}) / q$. Is this also a necessary condition? Note that there are only a countable number of local rules of type 2 , hence only a countable number of sets $\overline{\bar{t}_{t}}$ can admit local rules of type 2 .

Conjecture. If $\overline{\mathscr{T}_{t}}$ admits a local rule of type 2 , then $t=(m+n \sqrt{5}) / q$ for some integers $m, n, q$.

Note also that Theorem 4.7 is much stronger than Theorem 4.1. Every colored tiling satisfying $\mathscr{B}_{t}$ is a quasi-periodic colored tiling. That is, together with colors this tiling is still quasi-periodic, since it is the slice of a periodic colored tiling of $\mathbb{R}^{4}$.

\subsection{On Coloring}

When $t=(m+n \sqrt{5}) / q$ but is not of the form $m^{\prime}+n^{\prime} \tau$, then the set $\overline{\mathscr{T}}_{t}$ admits local rules of type 2 but does not admit local rules of type 1. Proposition 5.2 means that in this case the coloring is not a "local" operation.

We noted in the Introduction that, for such $t, T \in \overline{\mathscr{T}}_{t}$ exist which have two different colorings such that both of the colored tilings we obtain satisfy the local rule $\mathscr{B}_{t}$. They can be constructed as follows. Take a point $\alpha$ belonging to $\mathbf{p}^{\perp}\left(\mathbf{B}\left(\mathscr{O}_{t}^{\mathrm{c}}\right)\right)$ but not belonging to $\mathbf{p}^{\perp}\left(\mathbf{B}_{t}\right)$. Such a point always exists in this case. Then $\alpha$ is regular, but $\mathbf{E}+\alpha$ meets the parallel boundary of the refinement $\mathscr{O}_{t}^{\mathrm{c}}$. There are several lines in $\mathbf{p}^{\perp}\left(\mathbf{B}\left(\mathscr{O}_{t}^{\mathrm{c}}\right)\right)$ going through $\alpha$, they divide $\overline{\mathbf{E}}_{t}$ into many parts. Each part defines a colored tiling in $\overline{\mathscr{T}}\left(\mathscr{C}_{t}^{\mathrm{c}}\right)$ by taking the limit as in Section 2.5. Different parts define different colored tilings, but these tiling, when ignoring the color, are coincident with $T_{\alpha}$. It follows from the proof that only those tiling described above can be colored in different ways. Hence there is a subset of measure 0 in $\overline{\mathscr{T}}_{t}$ whose tilings can be colored in different ways, other tilings can be colored uniquely. $\overline{\mathscr{R}}\left(\mathbb{O}_{t}^{\mathrm{c}}\right)$.

When $t=m+n \tau$, each tiling $T \in \overline{\mathscr{T}}_{t}$ has a unique coloring as a colored tiling in

\subsection{Generalizations}

Our method can be applied to more general cases. First we introduce the following local rule of type 3 which contains both types 1 and 2 as special cases. Let a local rule of type 3 of radius $r$ be any finite set of colored $r$-maps. A noncolored tiling satisfies this local rule if it can be colored so that every colored $r$-map of the colored tiling is congruent to a colored $r$-map in this set.

Suppose $\mathbf{E}$ is a quadratic and totally irrational $n$-plane in the Euclidean space $\mathbb{R}^{N}$, equipped with a standard base. Here "quadratic" means that $\mathbf{E}$ is spanned by $n$ vectors $v_{1}, \ldots, v_{n}$ with coordinates belonging to $\mathbb{Z} \sqrt{d}$ for some natural number $d$, and "totally irrational" means that $\mathbf{E}$ des not contain any rational point except $\mathbf{0}$. Let $\overline{\mathbf{E}}$ be the $n$-plane spanned by $n$ vectors which are the conjugates of $v_{1}, \ldots, v_{n}$. Then $\mathbf{E} \cap \overline{\mathbf{E}}=\{0\}$ and $\mathbb{R}^{N}=\mathbf{E} \oplus \overline{\mathbf{E}} \oplus \Delta$ where $\Delta$ is a rational $(N-2 n)$-plane. For each 
$t \in \Delta$ let $\overline{\mathscr{T}}_{t}$ be the closure (under the operation "limit") of the set of all tilings $T_{\alpha}$ and their translates, where $\alpha$ is regular and belongs to $\overline{\mathbf{E}}+t$.

Theorem 7.1. If thas coordinates lying in $\mathbb{Q} \sqrt{d}$, then $\overline{\mathscr{T}_{t}}$ admits a local rule of type 3 .

This is a generalization of Theorem 4.1 to higher-dimensional cases, the proof is essentially the same. As for local rules of type 1, the situation is more complicated because of the bootstrapped condition. Some necessary conditions for the existence of local rules of type 1 are presented in [Lev] and [Le1].

\subsection{The Case $t=\frac{1}{2}$}

We present here a local rule of type 3 for the case $t=\frac{1}{2}$. This case enjoy as much symmetry as the case $t=0$ : both have the dihedral group $D_{5}$ as the symmetry group in the sense that $D_{5}$ preserves the same local isomorphism class. However, the latter has a local rule of type 1 while the case $t=\frac{1}{2}$ does not.

In order to reduce the number of prototiles we have to refine the proof of Theorem 4.7. The price for this is that we do not get a local rule of type 2 , but we get a local rule (of type 3 ) in which star-configurations play the role of edge-configurations.

Consider the six colored rhombs in Fig. 12(a). A tiling of the plane by images of the six colored rhombs under isometries of the plane (reflections are allowed) is said
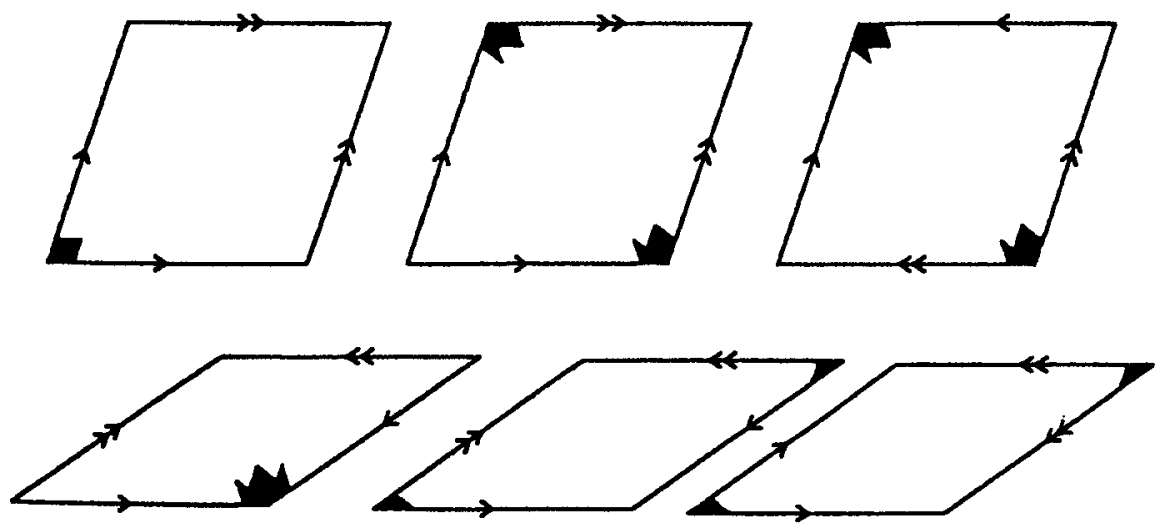

(a)
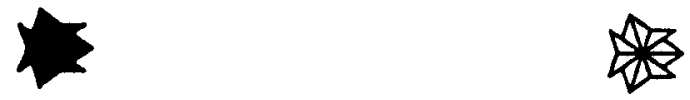

(b)

Fig. 12. Local rule for $t=\frac{1}{2}$. 
to be admissible if arrows on edges and marks at vertices are matched. Here matching at edges means every edge has a definite arrow, and matching at vertices means only marked vertices can meet and at every marked vertex the marks from all the rhombs incident to this vertex form a "key" like in Fig. 12(b).

The claim is that every admissible tiling, after some rotation, belongs to $\overline{\mathscr{T}}_{1 / 2}$. Conversely, every tiling in $\overline{\mathscr{T}}_{1 / 2}$ can be colored to become an admissible tiling.

Of course this is a local rule of type 3. This looks similar to the Ammann matching rules for the set of tilings having eight-fold symmetry (see [AGS] and [Le2]) and Socolar local rule for the set of tilings having twelve-fold symmetry [So1]. However, the methods to derive them are quite different. If we ignore the marks at vertices we get the Kleman-Pavlovitch matching rule (see [KP]).

There is a subset of measure 0 in $\overline{\mathscr{T}}_{1 / 2}$ such that every tiling in this subset can be colored to become an admissible tiling in two or four different ways, while every other tiling can be colored in a unique way. The key of Fig. 12(b) can appear in ten directions.

Remark. J. Socolar suggests that there is an interpretation of the vertex key in terms of Ammann's lines as in the cases of eight-fold and twelve-fold tilings (see [So1]).

Here is a sketch of the way we arrive at this local rule. First we refine the oblique periodic tiling $\mathscr{Q}_{1 / 2}$ to the oblique periodic colored tiling $\mathscr{O}_{1 / 2}^{\mathrm{cc}}$ whose parallel boundary contains all the five sets $\mathscr{F}-\varepsilon_{i} / 2, i=0, \ldots, 4$. Geometrically the refinement looks as follows. Every tile of $\mathscr{O}_{1 / 2}$ is the sum of a rhomb in $\mathbf{E}$ and a polygon in $\overline{\mathbf{E}}_{1 / 2}$. The latter is either a triangle or a hexagon; each hexagon has two long and one short diagonals. The refinement corresponds to dividing the hexagon into four parts by the two long diagonals and keeping all the triangles untouched. This refinement defines a set of colored tilings $\overline{\mathscr{T}}\left(\mathscr{O}_{1 / 2}^{c c}\right)$.

The following fact, which relates admissible tilings to the refinement, can be verified by analyzing the vertex-configuration of colored tilings in $\overline{\mathscr{T}}\left(\mathscr{O}_{1 / 2}^{\mathrm{cc}}\right)$ and by inspection.

Proposition 7.2. Every admissible tiling is a colored tilings whose vertex-configurations are translates of vertex-configurations of a colored tiling in $\overline{\mathscr{Y}}\left(\mathscr{Q}_{1 / 2}^{c c}\right)$.

Up to isometry and colors there are two rhombs: a thick one and a thin one. For a colored tiling let the thick part be the union of all the thick tiles. The following is trivial:

Lemma 7.3. If two admissible tilings are coincident on the thick part, they they are the same.

We consider the 2-planes in $\mathscr{F}-\varepsilon_{i} / 2$ as the big wall of $\mathscr{O}_{1 / 2}^{\text {cc }}$; all of them have width 0 . We call a tile of $\mathscr{G}_{1 / 2}$ a thick tile if its projection on $\mathbf{E}$ is a thick rhombs. 
Note that the refinement $\mathscr{G}_{1 / 2}^{\mathrm{cc}}$ is weaker than the refinement $\mathscr{O}_{1 / 2}^{\mathrm{c}}$ in Section 4. Now Proposition 4.6 is not valid for $\sigma_{1 / 2}^{c c}$ but it is still valid if the small wall $w$ projects onto a thick rhomb in $\mathbf{E}$ :

Lemma 7.4. The parallel boundary of $\mathscr{O}_{1 / 2}^{\mathrm{cc}}$ contains all the 2-plane from $\cup_{i=0}^{4}(\mathscr{F}-$ $\left.\varepsilon_{i} / 2\right)$. If the projection on $\mathbf{E}^{\perp}$ of a small wall $w$ of a thick tile of $\mathscr{\sigma}_{1 / 2}$ lies on the projection of a 2-plane $F$ from $\bigcup_{i=0}^{4}\left(\mathscr{F}-\varepsilon_{i} / 2\right)$, then $w$ and $F$ have nonempty intersection.

The lemma is proved easily by considering all the thick tiles of $\mathscr{O}_{1 / 2}^{\mathrm{cc}}$ which are finite up to translations.

Now suppose $T$ is a colored tilings whose vertex-configurations are the same as those of a tiling of $\overline{\mathscr{T}}\left(\mathscr{O}_{1 / 2}^{c c}\right)$. Then $T$ has a strongly connected lift into $\mathscr{O}_{1 / 2}^{\mathrm{cc}}$. Let $\rho$ be the multivalued mapping defined as in Section 6.2. If $F$ is a 2-plane from $\cup_{i=0}^{4}(F-$ $\varepsilon_{i} / 2$ ), then $\rho\left(\mathrm{p}(F)\right.$ ) does not intersect the line $\mathrm{p}^{\perp}(F)$ (see Proposition 6.4). The proof of Proposition 6.4 does not go through here (since the width $d$ of the big wall is 0 ), but this follows easily from the fact that not only edge-configurations but also the vertex-configurations of $T$ are the same as those of colored tilings of $\overline{\mathscr{T}}\left(\mathscr{O}_{1 / 2}^{\mathrm{cc}}\right)$. The zero width of the big wall is the reason why we have to use vertex-configuration instead of edge-configuration.

Hence we can orient the line $\mathbf{p}^{\perp}(F)$ such that $\rho\left(\mathbf{p}^{\perp}(F)\right)$ lies in the positive half-plane.

Note that the set $U_{i=0}^{4}\left(\mathscr{F}-\varepsilon_{i} / 2\right)$ does not have the bootstrapped property as formulated in Section 6.4, but it does satisfy the following weaker version: for every two parallel lines $h_{1}, h_{2}$ in $\mathbf{p}^{i}\left(\bigcup_{i=0}^{4}\left(\mathscr{F}-\varepsilon_{i} / 2\right)\right)$ there are two sequences of distinct lines $l_{i}, l_{i}^{\prime}, i \in \mathbb{Z}$ in $\mathbf{p}^{\perp}\left(\bigcup_{i=0}^{4}\left(\mathscr{F}-\varepsilon_{i} / 2\right)\right)$ such that:

(a) All lines $l_{i}$ are parallel, all lines $l_{i}^{\prime}$ are parallel, none of them is parallel to either of $h_{1}, h_{2}$.

(b) $l_{i}, l_{i}^{\prime}$, and $h_{1}$ have a common point and $l_{i}, l_{i+1}^{\prime}$ have a common point for every $i \in \mathbb{Z}$.

Now the proof of Theorem 4.1 shows that there is a colored tiling $T^{\prime}$ of $\overline{\mathcal{F}}\left(\mathscr{O}_{1 / 2}^{\mathrm{cc}}\right)$ such that $T=T^{\prime}$ on the thick part. Lemma 7.3 says that $T$ and $T^{\prime}$ are the same. This means every admissible tiling belongs to $\overline{\mathscr{P}}\left(\mathscr{O}_{1 / 2}^{\mathrm{cc}}\right)$.

\section{Acknowledgments}

The author is sincerely grateful to his adviser, Professor S. P. Novikov for attention to this work. He would like ot thank L. Danzer, C. Radin, and V. Sadov for fruitful discussions. He is especially indebted to $\mathrm{M}$. Senechal and J. Socolar who carefully checked the manuscript and suggested many things for the revised version. The author acknowledges the Max-Planck Institut für Mathematik for hospitality and support. 


\section{Appendix}

We prove Proposition 6.8. This proof is a modification of an (unpublished) proof of Levitov. The author learned of this proof through V. Sadov, but the proof that he learned from V. Sadov (for the class of quasi-crystals having eight-fold symmetry) contains a gap. In what follows we overcome the mistake and at the same time apply the proof to our cases.

Lemma A.1. For every two points $x, y \in \mathbf{E}$, if $|x-y|<1$, then the Hausdorff distance between $\rho(x)$ and $\rho(y)$ is less than a number $c_{1}$, not depending on $x$ and $y$.

Proof. This follows form the finiteness of the prototiles of $U$.

Lemma A.2. There is a constant $c_{2}$ such that for every line $h$ parallel to $p(F)$ where $F$ is a 2-plane from $\mathscr{F}$, the set $\rho(h)$ is contained in the $c_{2}$-neighborhood of a line $h^{\prime}$ parallel to $\mathbf{p}^{\perp}(F)$.

Proof. Suppose $F \subset \mathscr{F}_{0}$. Let $V$ be the set $\mathbf{p}^{\perp}\left(\mathscr{F}_{0} \cap \mathbf{p}^{-1}\left(h+U_{1 / 2}\right)\right)$. This set $V$ is a discrete family of lines parallel to $\mathbf{p}^{\perp}(F)$. There is a constant $d$ such that the least distance between two lines in $V$ is greater than $d$. The set $X=\rho\left(x+U_{1 / 2}\right)$, where $x$ is a point of $h$, is bounded, and the diameter of $X$ is less than $2 c_{1}$. If $H$ is a 2-plane in $\mathscr{F}_{0}$ such that $\mathrm{p}(H)$ intersects $x+U_{r}$, then there is a point of $X$ greater than $\mathbf{p}^{\perp}(H)$. Hence if $X$ does not meets $\mathbf{p}^{\perp}(H)$, then $X$ is greater than $\mathbf{p}^{\perp}(H)$.

Let $h_{1}^{\prime}, h_{2}^{\prime}$ be two lines from $V$ such that $X$ lies between them and every line from $V$ lying between them must intersect $X$. Then the distance between $h_{1}^{\prime}$ and $h^{\prime}{ }_{2}$ is less than $2 c_{1}+2 d$ (see Fig. 13).

Suppose $H_{1}, H_{2}$ are 2-planes from $\mathscr{F}_{0}$ such that $\mathbf{p}^{\perp}\left(H_{1}\right)=h_{1}^{\prime}, \mathbf{p}^{\perp}\left(H_{2}\right)=h_{2}^{\prime}$. Let $h_{1}=\mathbf{p}\left(H_{1}\right)$ and $h_{1}=\mathbf{p}\left(H_{2}\right)$.

By definition $\rho\left(h_{1}\right)>h_{1}^{\prime}, \rho\left(h_{2}\right)>h_{2}^{\prime}$. Since the distance between $h$ and $h_{1}$ (or $h_{2}$ ) is less than $\frac{1}{2}$, from Lemma A. 1 it follows that $\rho(h)$ lies between two lines $l_{1}$ and $l_{2}$ where $l_{1}$ (resp. $l_{2}$ ) is the line in $\overrightarrow{\mathbf{E}}$ parallel to $h_{1}^{\prime}$ and lying in the nonpositive half-plane of $h_{1}^{\prime}$ (resp. $h_{2}^{\prime}$ ) and having distance to $h_{1}^{\prime}$ (resp. $h_{2}^{\prime}$ ) equal to $c_{1}$. The distance between $l_{1}$ and $l_{2}$ is less than $c_{2}=3 c_{1}+2 d$.

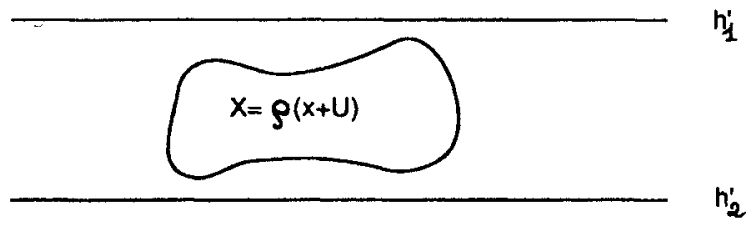

Fig. 13. Proof of Lemma A.2. 
Proof of Boundedness of $\boldsymbol{\rho}(\mathbf{E})$. Choose the coordinate systems $\left(a_{1}, a_{2}\right)$ in $\mathbf{E}$ and $\left(b_{1}, b_{2}\right)$ in $\overline{\mathbf{E}}$ such that $f_{1}$ is given by $\left\{a_{1}=0\right\}, f_{2}$ by $\left\{a_{2}=0\right\}, f_{4}$ by $\left\{a_{1}-a_{2}=0\right\}, \bar{f}_{1}$ is given by $\left\{b_{1}=0\right\}, \bar{f}_{2}$ by $\left\{b_{2}=0\right\}, \bar{f}_{4}$ by $\left\{b_{1}-b_{2}=0\right\}$. $0\}$.

Then it can be checked that $f_{3}$ is given by $\left\{a_{1}+\tau a_{2}=0\right\}$ and $\bar{f}_{3}$ by $\left\{\tau b_{1}+b_{2}=\right.$

For a pair of functions $\mu$ and $\nu$ on $\mathbf{E}$, let the sign $\mu \equiv \nu$ mean that $|\mu-\nu|<$ const. In other words, the distance between $\mu(x)$ and $\nu(x)$ is less than a constant for every $x \in \mathbf{E}$. If $\mu$ is a multivalued function on $\mathbf{E}$ (i.e., $\mu(x)$ is a subset of $\mathbb{R}$ ), then the distance between $\mu(x)$ and $\nu(x)$ is understood as the Hausdorff distance.

Note that $\rho$ is a multivalued map from $\mathbf{E}$ to $\overline{\mathbf{E}}$. Put

$$
\begin{aligned}
& b_{1}(x)=\left\{b_{1} \mid \exists b_{2} \text { such that }\left(b_{1}, b_{2}\right) \in \rho(x)\right\}, \\
& b_{2}(x)=\left\{b_{2} \mid \exists b_{1} \text { such that }\left(b_{1}, b_{2}\right) \in \rho(x)\right\} .
\end{aligned}
$$

Both $b_{1}(x), b_{2}(x)$ are multivalued function on E. By applying Lemma A.2 to lines parallel to $f_{1}$ we see that $b_{1}\left(a_{1}, a_{2}\right) \equiv \mu\left(a_{1}\right)$ for some function $\mu$ on $\mathbf{E}$, depending only on $a_{1}$, and hence can be regarded as a function on $\mathbb{R}$. Similarly $b_{2}\left(a_{1}, a_{2}\right) \equiv$ $\nu\left(a_{2}\right)$. By applying Lemma A.2 to $f_{4}$ we have $\mu\left(a_{1}\right)-\nu\left(a_{2}\right) \equiv \sigma\left(a_{1}-a_{2}\right)$ for some function $\sigma$.

Putting $a_{2}=0$ we see that $\mu \equiv \sigma$. Putting $a_{1}=0$ we get $\nu \equiv \sigma$. Hence $\mu \equiv \nu \equiv$ $\sigma$.

In addition $\mu$ also satisfies Lemma A.1. From this it is not difficult to prove that for a fixed constant $c$ we have $\mu(c x) \equiv c \mu(x)$.

Finally, by applying Lemma A.2 to $f_{3}$, we have $\mu\left(a_{1}\right)+\tau \mu\left(a_{2}\right) \equiv \theta\left(\tau a_{1}+a_{2}\right)$ for some function $\theta$; putting $a_{2}=0$ we get $\mu \equiv \theta$. Then

$$
(1-\tau) \mu\left(a_{1}\right) \equiv(\tau-1) \mu\left(a_{2}\right) \text {. }
$$

Because $\tau \neq 1$ (this is the expression of "completeness" defined in [LPS1] and [Le1]) it follows that $\mu \equiv 0$. This means that $\rho$ is bounded.

Boundedness of $\mathbf{p}^{\perp}(\rho(\mathbf{E}))$ means the local rule is a "weak local rule" in the sense of Levitov. For every $t \in \mathbb{R}$ one can easily construct a local rule of type 1 such that every tiling satisfying this local rule has a strongly connected lift into $\mathscr{\theta}_{t}$. Using a similar proof as the above it can be proved that this local rule is a "weak local rule" in the sense of Levitov. A similar method works in the higher-dimensional case as well. The existence of weak local rules for $\overline{\mathscr{F}}_{t}$, has also been proved by Levitov [Lev] by a different method.

\section{References}

[AGS] R. Ammann, B. Grünbaum, and G. C. Shephard, Aperiodic tiles, Discrete Comput. Geom., 8 (1992), 1-25.

[dB1] N. G. de Bruijn, Algebraic theory of Penrose non-periodic tilings, Nederl. Akad. Wetensch. Proc. Ser. A, 84 (1981), 39-66. 
[dB2] N. G. de Bruijn, On Beenker patterns, Preprint, 1991.

[B] S. E. Burkov, Absence of weak local rules for the planar quasicrystalline tiling with 8-fold symmetry, Comm. Math. Phys., 119 (1988), 667-675.

[D1] L. Danzer, Three-dimensional analog of the planar Penrose tilings and quasicrystals, Discrete Math., 76 (1989), 1-7.

[D2] L. Danzer, Quasiperiodicity; local and global aspects, in Group Theoretical Methods in Physics, Lecture Notes in Physics, Springer-Verlag, Berlin, 1991.

[GR] F. Gahler and J. Rhyner, Equivalence of the generalized grid and projection methods for the construction of quasiperiodic tilings, J. Phys. A, 19 (1986), 267-277.

[IS] K. Ingersent and P. Steinhardt, Matching rules and growth rules for pentagonal quasicrystal tilings, Preprint, 1991.

[K] A. Katz, Theory of matching rules for 3-dimensional Penrose tilings, Comm. Math. Phys., 119 (1988), 262-288.

[KP] M. Kleman and A. Pavlovitch, Generalized 2D Penrose tilings: structural properties, J. Phys. $A, 20$ (1987), 687-702.

[Lev] L. S. Levitov, Local rules for quasicrystals, Comm. Math. Phys., 119 (1988), 627-666.

[Le1] T. Q. T. Le, Necessary conditions for the existence of local rules for quasicrystals, Preprint MPI/92-98, Max-Planck Institut für Mathematik, Bonn, 1992.

[Le2] T. Q. T. Le, Local rules for planar quasiperiodic tilings having 8-fold symmetry, Preprint MPI/92-102, Max-Planck Institut für Mathematik, Bonn, 1992.

[LPS1] T. Q. T. Le, S. Piunikhin, and V. Sadov, Local rules for quasiperiodic tilings of quadratic 2-planes in $\mathbb{R}^{4}$, Comm. Math. Phys., 150 (1992), $23-44$.

[LPS2] T. Q. T. Le, S. Piunikhin, and V. Sadov, Geometry of quasicrystals, Uspekhi Mat. Nauk., 48 (1993), 41-102 (in Russian). English translation: Russian Math. Surveys, 48 (1993), 37-100.

[ODK] C. Oguey, M. Duneau, and A. Katz, A geometrical approach to quasiperiodic tilings, Comm. Math. Phys., 118 (1988), 99-118.

[P] R. Penrose, Tilings and quasicrystals; a non-local problem?, in Aperiodicity and Order, Vol. 2, ed. by M. V. Jarić, Academic Press, New York, 1989.

[Ral] C. Radin, Global order from local sources, Bull. Amer. Math. Soc., 25 (1991), 335-364.

[Ra2] C. Radin, The pinwheel tilings of the plane, Ann. of Math., to appear.

[Ro] A. Robinson, The dynamical theory of tilings and quasicrystallography, Preprint, George Washington University, 1992.

[Se] M. Senechal, Quasicrystals and Geometry, Cambridge University Press, Cambridge, to appear.

[So1] J. Socolar, Simple octagonal and dodecagonal quasicrystals, Phys. Rev. B, 39 (1989), 10519-10551.

[So2] J. Socolar, Weak matching rules for quasicrystals, Comm. Math. Phys., 129 (1990), 599-619.

Received March 1, 1994, and in revised form September 29, 1994. 